Meta

Journal des traducteurs

Translators' Journal

\title{
Je construis ma maison. Étude lexico-terminologique de la construction
}

\author{
Cécile Bruneau, Marina de Almeida, Margreet de Rooij, Marie-Hélène Lattaro, \\ Mayra Parra, Justine Ravonihasindaza et Hélène Saulnier
}

Volume 42, numéro 4, décembre 1997

URI : https://id.erudit.org/iderudit/002151ar

DOI : https://doi.org/10.7202/002151ar

Aller au sommaire du numéro

Éditeur(s)

Les Presses de l'Université de Montréal

ISSN

0026-0452 (imprimé)

1492-1421 (numérique)

Découvrir la revue

Citer cet article

Bruneau, C., de Almeida, M., de Rooij, M., Lattaro, M.-H., Parra, M., Ravonihasindaza, J. \& Saulnier, H. (1997). Je construis ma maison. Étude lexico-terminologique de la construction. Meta, 42(4), 685-724.

https://doi.org/10.7202/002151ar
Résumé de l'article

Cet article présente les grandes étapes d'une recherche terminologique thématique portant sur la construction résidentielle - découpage du domaine, élaboration des arbres de domaines, recherche bibliographique, mise au point de la fiche terminologique, élaboration des définitions, etc. - effectuée par un groupe de sept étudiantes dans le cadre du cours "Terminologie théorique et pratique II" dispensé par le professeur André Cías à l'Université de Montréal. 


\title{
JE CONSTRUIS MA MAISON. ÉTUDE LEXICO-TERMINOLOGIQUE DE LA CONSTRUCTION
}

\author{
Résumé \\ Cet article présente les grandes étapes d'une recherche terminologique thématique \\ portant sur la construction résidentielle - découpage du domaine, élaboration des arbres de \\ domaines, recherche bibliographique, mise au point de la fiche terminologique, élaboration \\ des définitions, etc. - effectuée par un groupe de sept étudiantes dans le cadre du cours \\ "Terminologie théorique et pratique II» dispensé par le professeur André Clas à l' Université \\ de Montréal.
}

\begin{abstract}
A group of graduate students in the "Terminologie théorie et pratique II" seminar conducted by Professor André Clas at Université de Montréal presents the main steps involved in a thematic terminological research on residential construction: domain specification, field trees determination, bibliographical researchs, establishment of terminological records, creation of definitions, etc.
\end{abstract}

\section{INTRODUCTION}

Avec la crise de 1929, l'industrie de la construction, au Canada, connaît une forte baisse. Par contre, la Seconde Guerre mondiale relance cette activité, avec la réalisation de grands projets tels une usine de caoutchouc synthétique à Sarnia, en Ontario, de nombreux aérodromes et des installations militaires. Après la guerre, les besoins en logement augmentent et l'on voit apparaître de nombreux projets domiciliaires. Avec l'avènement de nouvelles techniques et de nouvelles normes, le domaine du bâtiment connaît une véritable révolution et une grande partie de ses activités s'industrialise. $\mathrm{Au}$ début des années 1980, les projets de construction gouvernementaux s'élèvent annuellement à près de 60 milliards de dollars et l'industrie de la construction fournit un emploi à 750000 personnes par année. De plus, des programmes gouvernementaux stimulent la construction dans le secteur résidentiel et, encore aujourd'hui, le Programme d'accès à la propriété permet chaque année à de nombreuses personnes de faire construire leur maison.

L'industrie de la construction est un domaine extrêmement vaste qui touche presque tous les secteurs de la vie: droit, urbanisme, architecture, outillage, isolation, électricité, matériaux, etc. Sa terminologie est donc très variée. De plus, il s'agit d'une industrie assez conservatrice dont le vocabulaire est empreint de termes des méthodes de construction artisanales, qui varient selon les régions, et de termes techniques des méthodes nouvelles.

\section{MÉTHODOLOGIE GÉNÉRALE}

Avant d'exposer la méthodologie générale adoptée par le groupe, il semble intéressant de faire une remarque. On note que le franco-français emploie le terme bâtiment pour décrire cette industrie, alors que le franco-québécois privilégie le terme de construction. Existe-t-il une nuance entre les deux termes, tout comme entre bâtir et construire, entre bâtisseur et constructeur? La construction se réfère à l'ensemble des techniques qui permettent de construire, de bâtir (Nouveau Petit Robert), alors que le bâtiment correspond, par extension, à l'ensemble des industries et des métiers qui concourent à la construction des édifices. Le terme construction est donc plus générique. Par contre, on note l'inverse lorsqu'il s'agit des verbes. En effet, construire c'est bâtir selon un plan déterminé, alors que bâtir c'est élever sur le sol. Le premier semble donc plus spécifique que le second. De plus, le verbe bâtir paraît plus vieux, plus archaïque que le verbe construire. Il revêt aussi la connotation d'effort. Finalement, les termes bâtisseur et constructeur ont tous deux le sens figuré de fondateur; cependant, dans le 
domaine de la construction, on parle plus d'un constructeur que d'un bâtisseur. Qu'en est-il alors des équivalents anglais? Selon les définitions anglaises, il ne semble pas y avoir de différence entre construction et building et entre constructor et builder. Quant à la nuance existant entre build et construct, elle n'est pas claire. Le premier verbe semble simplement être plus employé et avoir évolué davantage que le second.

Étant donné que le domaine de la construction est si vaste, comprenant des aspects tant juridiques et architecturaux que géologiques et techniques, nous avons limité notre choix à la construction d'une maison. Nous nous sommes concentrées sur les quatre sousdomaines suivants: les matériaux (Cécile Bruneau et Marina de Almeida), la maçonnerie (Margreet de Rooij et Mayra Parra), la toiture (Marie-Hélène Lattaro et Justine Ravonihasindaza) et les ouvertures (Hélène Saulnier). Il s'agit d'un choix arbitraire qui, à notre avis, représente cependant les aspects essentiels liés à la construction d'une maison.

Afin de garantir la cohérence du travail, nous avons élaboré un modèle de fiche terminologique. Les champs suivants ont été retenus: l'entrée, les synonymes (le cas échéant), la catégorie grammaticale, le ou les équivalents anglais, la définition et/ou le contexte, des observations (éventuellement), la source de la définition (en indiquant le nom de l'auteur et la date), le sous-domaine et l'auteur de la fiche. Les synonymes français font l'objet d'une entrée séparée avec un renvoi au terme principal. Nous avons relevé les diverses définitions proposées par les auteurs, que ce soit dans les dictionnaires ou dans les monographies, mais nous avons décidé autant que possible d'établir nos propres définitions pour chacun des termes sélectionnés. Cependant, dans le cas où la définition proposée par un des auteurs décrit parfaitement la notion, elle est conservée telle quelle.

Nous nous sommes vite aperçues du grand nombre d'ouvrages publiés dans le domaine de la construction, et des critères de sélection se sont donc imposés. Les critères suivants ont été retenus :

- Date de publication - le domaine de la construction est en évolution constante, c'est pourquoi nous avons choisi les ouvrages les plus récents dont le contenu rendait compte des nouvelles technologies.

- Auteur et éditeur - un auteur qui a publié plusieurs livres, qui est cité dans d'autres ouvrages ou qui apparaît dans la bibliographie d'ouvrages du même domaine, peut être considéré comme une autorité dans la matière. Une maison d'édition spécialisée peut être une autre indication de fiabilité.

- Réédition de l'ouvrage - si un ouvrage a connu plusieurs éditions, cela veut dire qu'il a eu du succès et par conséquent qu'il est fiable, surtout lorsque la nouvelle édition indique que l'ouvrage a été revu et augmenté.

- Index et bibliographie - la présence d'un index et d'une bibliographie augmente la crédibilité de l'ouvrage, car cela implique que l'auteur a fait des recherches. Pour le terminologue, l'index est précieux, puisqu'il facilite la tâche de recherche.

- Ouvrage non traduit - un ouvrage en langue originale est, en principe, toujours plus proche de la réalité de cette langue qu'un ouvrage traduit.

Une fois le travail divisé et les critères établis, chaque équipe a continué la recherche documentaire, se consacrant à son domaine spécifique. Dans la suite de l'article, chaque équipe présente son cheminement et les problèmes qu'elle a rencontrés.

\section{MATÉRIAUX}

Premièrement, nous avons tenté de dresser l'arbre du sous-domaine des matériaux. Pour ce faire, nous nous sommes basées sur deux ouvrages, le Dicobat, de Jean de Vigan (1992) et le Guide de la technique II - les matériaux (1991), publié par les Presses polytechniques et universitaires romandes. 
Dans notre premier arbre, nous avions mis, sous matériaux, quatre grandes catégories à un même niveau, à savoir les minéraux, les végétaux, les métaux et les matériaux préfabriqués. Chaque catégorie était subdivisée en type, propriété et travail. Lors du dépouillement et des lectures, nous nous sommes vite rendu compte qu'un tel arbre n'était pas satisfaisant. Il paraissait trop simpliste et redondant à la fois.

Pour établir le second arbre, nous avons essayé de découper le domaine plus finement. Sous les matériaux ( $1^{\mathrm{er}}$ niveau), nous avons opposé deux grandes catégories: minéraux et végétaux. Ensuite, sous minéraux ( $2^{\mathrm{e}}$ niveau), nous distinguions, d'une part, les métaux, et d'autre part, les minéraux non métalliques. Nous avons choisi de n'inclure dans les végétaux ( $2^{\mathrm{e}}$ niveau) que le bois de construction. Sous les métaux $\left(3^{\mathrm{e}}\right.$ niveau), nous traitions deux branches, les métaux de base et les alliages. La catégorie des minéraux non métalliques ( $3^{\mathrm{e}}$ niveau) se subdivisait en matériaux élaborés et en matériaux bruts. Finalement, nous avions décidé de traiter sous matériaux élaborés ( $4^{\mathrm{e}}$ niveau), les matériaux composites, d'une part, et les composantes, d'autre part. Quant aux matériaux bruts $\left(4^{\mathrm{e}}\right.$ niveau), ils comprenaient trois branches: les pierres/roches, les granulats et la marne. En sixième niveau, nous avions donné différents types de matériaux composites, de composantes et pierres/roches. Les différents niveaux se schématisaient comme suit:

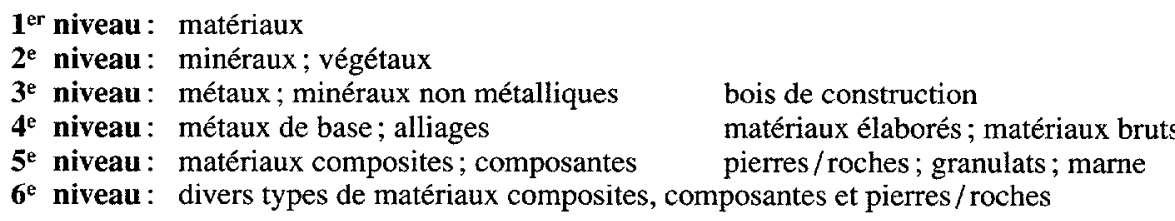

Cependant, ce découpage présentait encore quelques faiblesses. En effet, il semblait plus logique de répartir les minéraux en métaux et non métaux, tout comme les métaux en alliages et non alliages. Ensuite, nous avons éliminé la branche des matériaux composites et celle des composantes. En effet, on retrouvait des matériaux composites et des matériaux bruts dans la catégorie des composantes. Nous avons donc décidé de répartir ces éléments entre les matériaux élaborés et les matériaux bruts. Ainsi, adjuvant, chaux et plâtre, auparavant sous la catégorie composantes, appartiennent désormais à la branche des matériaux élaborés. De même, le gypse, le calcaire et l'argile, également des composantes, mais des matières retrouvées à l'état brut dans la nature, sont placées dans les matériaux bruts. Dans cette dernière catégorie, nous avons finalement décidé d'éliminer les pierres/roches et les granulats, car ceux-ci ne s'appliquaient pas à tout. La représentation finale de l'arbre des matériaux est la suivante :

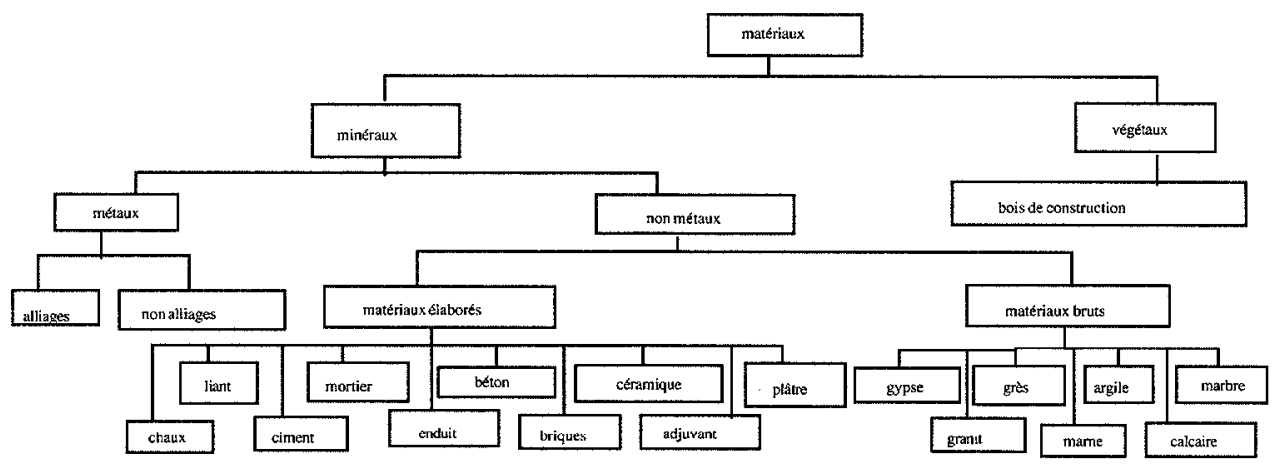


En ce qui concerne la méthodologie de recherche des termes, dans un premier temps, nous avons sélectionné les ouvrages qui nous serviraient à établir notre nomenclature. Il est intéressant de remarquer que nombre de ces ouvrages appartiennent au sousdomaine de la maçonnerie, celui-ci étant étroitement lié au nôtre. Après dépouillement du corpus tel qu'établi, nous avons décidé d'approfondir deux branches de l'arbre du domaine: celle des matériaux bruts et celle des matériaux élaborés. Nous avons donc relevé nos termes en fonction de ce choix. Le domaine des matériaux est très vaste et nous aurions pu traiter plus de 200 termes; cependant, dans les limites du présent travail et pour ne pas empiéter sur les autres sous-domaines, nous avons dû nous limiter à une soixantaine de termes. Nous avons cherché les équivalents en anglais pour chacun des termes choisis. Ces équivalents, relevés dans les lexiques et dictionnaires bilingues, ont été par la suite validés par une lecture d'ouvrages unilingues en langue anglaise. Nous avons tenté, lorsque le cas se présentait, de faire un tri entre les différents synonymes relevés pour les termes, en français comme en anglais. De même, lorsque nous avons rencontré des éléments encyclopédiques intéressants, ou si nous avons trouvé des collocations s'appliquant aux termes traités, nous les avons relevés en observation.

\section{MAÇONNERIE}

Les premières recherches nous ont amenées à faire une distinction entre la grosse maçonnerie, définie comme l'ensemble des ouvrages d'un bâtiment qui composent son ossature et assure la stabilité et la cohésion d'une construction, et la petite maçonnerie, à savoir l'ensemble des travaux et des ouvrages qui ne participent pas à la stabilité de la construction. Toutefois, ces termes ne sont pas utilisés dans le milieu, du moins pas au Québec, où l'on emploie plutôt maçonnerie structurelle et maçonnerie de finition, respectivement.

Le travail de maçonnerie étant réalisé essentiellement par le briqueteur-maçon, nous nous sommes basées sur les tâches de ce métier, décrites dans une brochure de la Commission de la Construction du Québec (CCQ). Selon cette brochure, les tâches du briqueteur-maçon sont plutôt de maçonnerie de finition. Quant à la maçonnerie structurelle, elle requiert aujourd'hui surtout le travail du charpentier-menuisier pour le coffrage et du cimentier-applicateur pour couler le béton.

Dans nos recherches, il est apparu qu'au sein de la maçonnerie de finition on peut distinguer des techniques (la pose de pierre, de brique, etc.), des matériaux (traités par une des autres équipes) et le matériel, qui est la branche que nous avons décidé d'approfondir.

Dans le matériel, on peut distinguer l'outil (tout objet fabriqué qui sert à exécuter un travail déterminé et qui agit sur la matière), l'instrument (objet fabriqué qui sert à exécuter une tâche) et l'équipement (vêtements et matériel adaptés à une activité particulière). L'équipement étant plutôt accessoire à l'exécution du travail proprement dit, nous ne l'avons pas retenu.

Nous avons constaté que les ouvrages consultés classaient les outils de différentes façons. Une première classification distingue quatre catégories d'outils, à savoir les outils manuels, électroportatifs, pneumatiques et à poste fixe. Le problème que nous avons rencontré avec cette façon de classer est que, par exemple, le marteau de maçon ferait partie des outils manuels, alors que le marteau pneumatique ferait partie des outils pneumatiques, les deux outils ayant pourtant une même fonction. Cela nous a amenées à répartir les outils selon leur fonction, à savoir outils coupants, d'assemblage, de frappe, de finition et de mélange. 


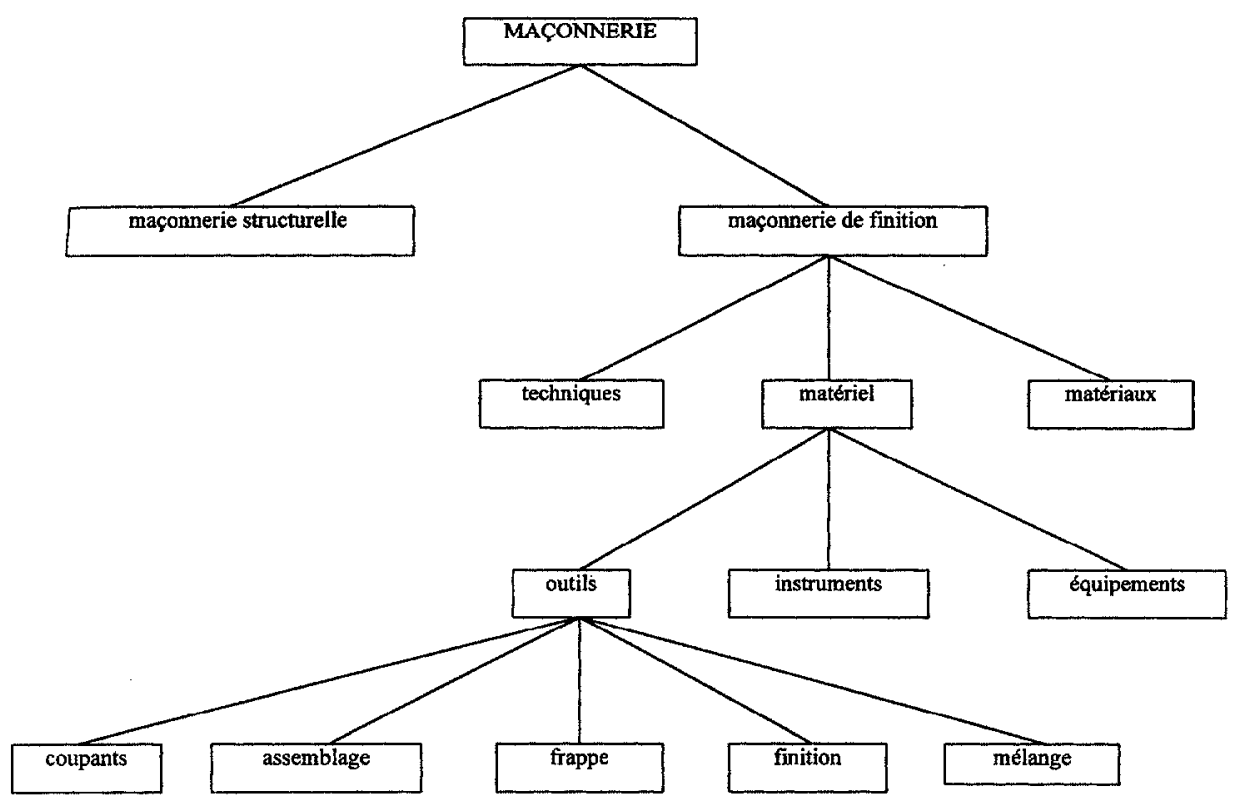

Pour déterminer le champ de la maçonnerie et les tâches du maçon, nous avons commencé par rassembler de la documentation sur le domaine, en anglais et en français. La première chose qui nous a sauté aux yeux est la différence dans la façon dont l'anglais et le français découpent le domaine ${ }^{1}$. Notre langue de départ étant le français, nous avons choisi de suivre la division coutumière dans cette langue. Ensuite, nous avons choisi la branche à traiter, à savoir celle du matériel utilisé dans le travail de maçonnerie.

L'étape suivante a été celle du dépouillement de la documentation spécialisée en anglais et en français, en triant les ouvrages trouvés selon les critères mentionnés plus haut. Chacune de nous s'est consacrée à une seule langue. Cette méthode a facilité la recherche, puisque chacune s'est familiarisée rapidement au langage du domaine, soit en anglais, soit en français, pour arriver ainsi plus rapidement à l'information recherchée. Cette information nous a menées à faire une première liste du matériel de maçonnerie.

Nous avons alors constaté que notre nomenclature comprenait trop de termes pour le cadre limité de cette étude. De plus, plusieurs termes nous semblaient synonymes, problème qui sera traité plus loin.

Les définitions trouvées dans les dictionnaires et ouvrages spécialisés se sont révélées insuffisantes pour le modèle de définition que nous nous sommes proposé, à savoir de décrire l'aspect physique de l'objet défini, ainsi que sa fonction.

Pour résoudre les problèmes de nomenclature et de définition, nous avons eu recours à l'aide d'un expert ${ }^{2}$. Il nous a indiqué quels étaient les instruments vraiment utilisés par le briqueteur-maçon au Québec, nous a aidées à compléter les définitions et à déterminer les termes synonymes, en précisant le terme le plus fréquent au Québec. Nous nous sommes aperçues qu'un grand nombre de termes relevés dans les ouvrages ne s'utilisent 
pas dans la pratique, ce qui, par ailleurs, est un phénomène courant dans le domaine technique. En dépit de cette différence, nous avons retenu les termes spécifiques les plus souvent rencontrés dans les ouvrages spécialisés.

Une fois la nomenclature en français établie, nous avons fait le crochet terminologique, c'est-à-dire la fusion des termes français et anglais, par comparaison des définitions dans les deux langues.

\section{OUVERTURES}

Lorsqu'on regarde une fenêtre, il nous est difficile d'imaginer la grande quantité de termes qui existent pour en nommer toutes les parties. Et pourtant, c'est le cas. Et ce qui est encore plus surprenant quand on aborde la terminologie de la fenêtre, c'est le temps que l'on peut passer à structurer le domaine, à construire l'arbre du domaine. Ça semble si simple une fenêtre! Eh bien, nous avons eu la preuve qu'en terminologie rien n'est simple...

Il a fallu d'abord déterminer le point de départ du domaine, le sommet de l'arbre. La fenêtre étant définie comme un ouvrage de menuiserie dans une baie, nous en avons conclu que baie serait notre point de départ: baie = ouverture. Il existe cependant différents types d'ouvertures dans une maison, notamment celles des portes et celles des fenêtres. Nous avons donc choisi de nous concentrer sur les baies de fenêtres. Ensuite, une baie comportant un encadrement, nous avons décidé d'en traiter les composantes. Ici encore, nous avons fait un choix, celui de ne traiter que les parties extérieures de la baie. Nous avons d'ailleurs fait le même choix pour le traitement de la fenêtre: nous nous sommes concentrées sur ses composantes extérieures. S'il arrive qu'un terme désigne à la fois une composante intérieure et extérieure, nous l'avons conservé. Au sommet de l'arbre se retrouvent donc la baie et ses composantes. Vient ensuite la fenêtre, ouvrage de menuiserie dans une baie. Traiter la fenêtre a été plus difficile, car il s'agit d'un ensemble complexe d'éléments, et, en effet, comment classer toutes ces informations?

Les points suivants ont été traités de façon plus détaillée : la baie et ses composantes, les types de fenêtres (selon le type d'ouverture), les composantes de la fenêtre (bâti dormant et châssis), les composantes du châssis et quelques éléments d'isolation de celui-ci. Il nous a semblé important de parler de l'isolation des fenêtres, préoccupation certaine au Canada, mais nous n'avons pas fait le tour du sujet, les éléments d'isolation étant des éléments du châssis participant à l'isolation. En traitant les composantes du châssis, nous n'avons pas retenu les différents types de verre qui représentent à eux seuls un sujet de recherche.

Il nous a aussi fallu dessiner les autres branches de l'arbre de domaine: les matériaux de fabrication des fenêtres (réalisé par une autre équipe); la position de la fenêtre sur la maison (toit, mur et façade, sous-sol); les organes de fermeture et de rotation dont nous n'avons retenu que paumelle, pivot et biellette, termes apparaissant dans les définitions des types de fenêtres; les fermetures extérieures comme les volets et contrevents. Nous croyons avoir fait, grâce à cet arbre, une exploration assez complète du domaine.

Notre recherche a commencé par une visite des bibliothèques (municipale, universitaire), afin de dresser une liste d'ouvrages anglais et français pertinents, grâce auxquels nous nous sommes constitué une «banque» de documents, dont une majorité est en français. Après avoir rassemblé les documents, nous avons lu, relu, souligné, et nous avons recensé tous les termes qui nous semblaient se rapporter aux fenêtres. Cette vision large nous a permis de comprendre le domaine, d'en faire le tour. Tout en faisant cela, nous essayions de commencer à esquisser un arbre pour éviter de nous attarder sur des 


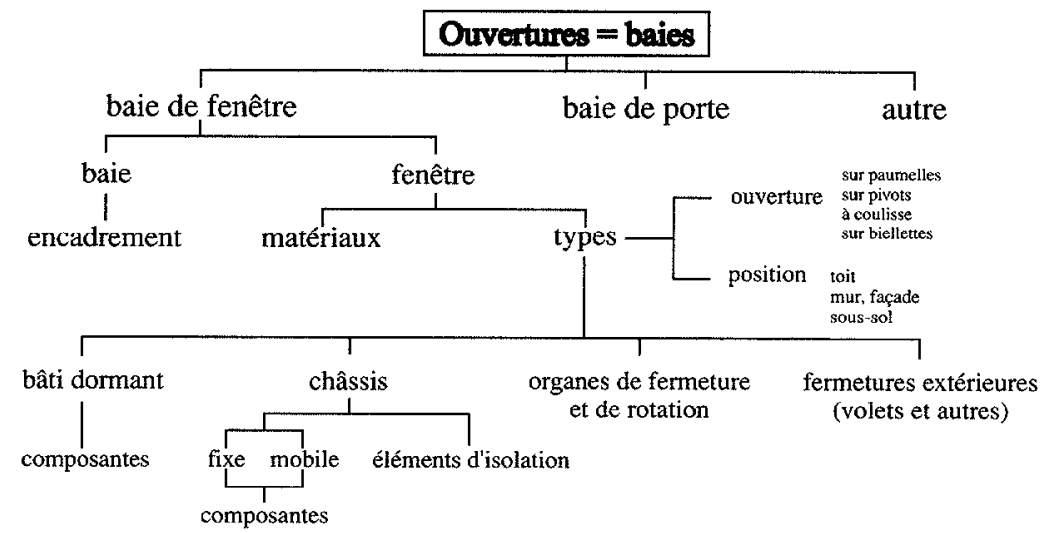

termes que finalement nous rejetterions. Notons, en passant, que nous avons construit l'arbre au fil de nos lectures, le modifiant jusqu'à la dernière minute. À mesure que nous avions une idée plus claire du sujet et une meilleure compréhension des définitions, la forme de l'arbre se précisait et certaines branches tombaient.

Une des difficultés que nous avons rencontrées a été de savoir quel terme ou quelle définition étaient utilisés au Québec plutôt qu'en France. Il nous a fallu garder cette préoccupation en mémoire tout au long de notre travail. Nous avons grappillé, au cours de nos visites chez les détaillants de fenêtres, des informations sur l'usage au Québec. C'est ainsi que nous avons découvert que les détaillants d'ici appellent volet un châssis.

Il se peut qu'il reste encore dans notre liste des termes davantage usités en France qu'au Québec, même si nous en avons éliminé plusieurs tels que fenêtre accordéon ou fenêtre carrousel. Aurions-nous dû éliminer aussi fenêtre à l'australienne, à l'italienne ? Seule une recherche plus poussée nous permettrait de répondre à cette question. Nous avons toutefois décidé de garder ces termes, car nous croyons qu'ils existent dans la réalité nord-américaine, beaucoup plus vaste que la réalité québécoise.

\section{TOITURE}

Notre première étape a consisté à recueillir toute la documentation nécessaire à notre secteur de recherche (qui était alors la charpente au sens large). Nous avons donc écumé bibliothèques et associations, et navigué sur Internet. Nous avons ainsi recueilli une foule de documents. En épluchant ces documents, nous avons découvert que les études sur les charpentes étaient soit succinctes, quoique souvent accompagnées de schémas clairs, soit exhaustives, surtout lorsqu'il s'agissait de guides de construction. Il a donc fallu étoffer les unes et dépouiller les autres.

La seconde étape a consisté à délimiter clairement notre domaine de recherche. Nous avons donc distingué, d'une part, la charpente de maison de la charpente de toiture et, d'autre part, le support de couverture de la charpente. Étant donné la très grande diversité des concepts et des termes reliés à la charpente, nous avons décidé de concentrer nos efforts sur la toiture, sa couverture et sa charpente. De plus, afin de rationaliser la structure globale de la charpente du toit et de sa couverture, nous avons décidé d'omettre tout détail s'écartant du minimum vital nécessaire pour la toiture d'une maison d'habitation. Ainsi, nous n'avons, par exemple, traité ni les systèmes d'étanchéité et d'isolation, ni les multiples ouvertures et installations possibles (lanterneaux, lucarnes, paratonnerres, etc.). 
En abordant les termes, à la troisième étape de notre démarche, nous avons noté, entre autres, que certains mots avaient un sens différent de celui du langage quotidien. Par exemple, alors que couramment, les combles se limitent à l'espace compris entre le toit et le plafond de l'étage supérieur d'une maison, son singulier indique, dans le domaine de la construction, l'ensemble de la superstructure comprenant la charpente et la couverture. Comme tête d'arbre, nous avons toutefois préféré toiture, à comble ou à toit, d'abord parce que c'est le terme choisi par l'Organisation Internationale de Normalisation (ISO), tant comme terme que comme cooccurrent (toiture inclinée, toiture à la Mansart, etc.), ensuite parce que le sens courant de comble semble vraiment l'emporter sur son sens «didactique».

Nous nous sommes ensuite rendues compte qu'il était difficile de parler de charpente et de couverture sans aborder les différents types de toitures... et nous avons ouvert la boîte de Pandore. Il existe en effet dans ce domaine le plus grand fouillis : les architectes de tous les temps s'en sont donné à cœur joie, si bien qu'il existe une telle diversité de formes de toitures (et, partant, une telle diversité de termes pour les désigner) qu'il est presque impossible d'en faire le tour. Nous avons donc délimité trois grandes catégories de toitures: les toitures plates (dont la charpente ressemble à celle des planchers), les toitures inclinées et les toitures arrondies (dont la charpente est très particulière). Nous avons choisi d'approfondir les branches relatives au type de toiture le plus courant, soit la toiture inclinée à plusieurs pentes (en prenant comme modèle la très classique toiture à pignons!).

La charpente de ces toitures peut être ou non composée de fermes. Parce que les charpentes à fermes sont les plus courantes, c'est ce type de charpente que nous avons étudié. Le type de la ferme dépend largement de la portée qu'elle doit couvrir : comme les grandes portées sont plutôt le fait des bâtiments industriels, nous avons étudié les fermes utilisées pour les petites et les moyennes portées, que l'on retrouve dans les maisons individuelles. Ces fermes se divisent en deux types: classique et moderne. Les fermes classiques sont celles que construisaient nos ancêtres charpentiers, à l'aide de grosses poutres de bois qu'ils assemblaient au moyen de techniques de charpenterie et de menuiserie (tenons et mortaises, chevilles de bois, etc.). Les fermes modernes sont celles que construisent, en usine, les techniciens d'aujourd'hui : elles ne sont plus composées de poutres, mais de pièces de bois de faible équarrissage ( 22 par $4 »)$ et d'assemblages métalliques (boulons, clous, connecteurs divers, etc.). Les calculs de triangulation remplacent l'intuition et l'expérience des charpentiers d'antan et permettent de créer des fermes solides, rendant possibles pratiquement toutes les fantaisies architecturales.

Sur la charpente repose la couverture, qui protège les espaces intérieurs contre les intempéries. Ses composantes principales sont le support de couverture et les matériaux de recouvrement. Les premiers, qui se trouvent directement sur la charpente, servent de support aux matériaux de couverture, qui assurent l'étanchéité de la toiture.

Bien qu'ils soient clairement divisés dans l'arbre, tous les éléments composant la toiture sont interdépendants. En effet, le support de couverture, défini ici uniquement comme structure porteuse des matériaux de couverture, contribue également à la rigidité de la charpente; le type de matériau de couverture choisi dépend largement de la pente du toit (ou l'inverse), laquelle détermine la structure de la ferme; de la pente du toit et de la portée dépendent le nombre de fermes utilisées; etc. 

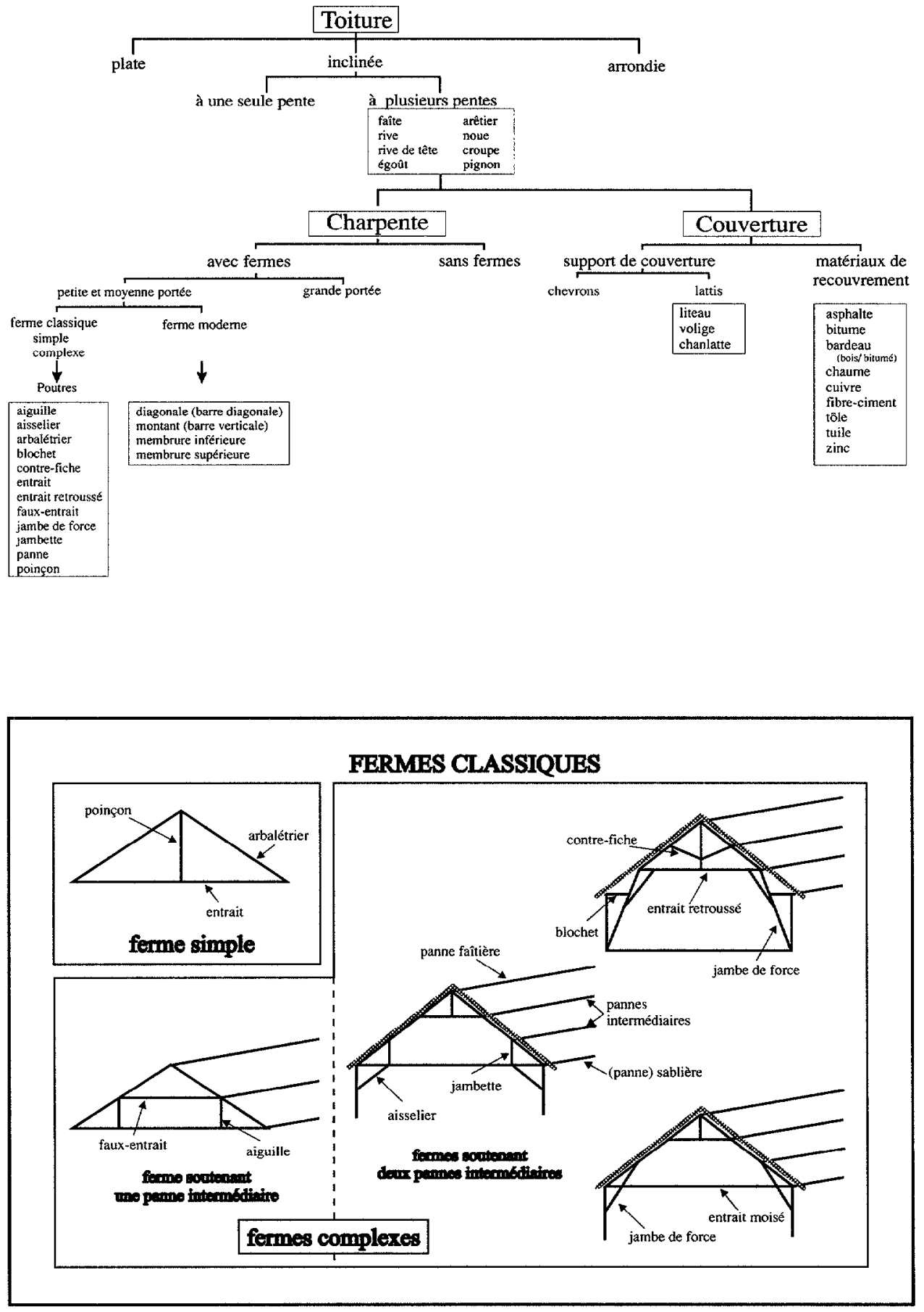
Pour conclure, quelques remarques s'imposent. À quelques reprises, nous avons remarqué que le même nom peut s'éloigner de son sens premier et même le contredire, lorsqu'on le combine à un autre. Par exemple, alors que la rive est la ligne délimitant divers plans avec le vide, on appelle rive de tête, le bord supérieur de la toiture à un seul versant d'une dépendance, qui reste pourtant accolé à la construction principale. Par ailleurs, les substantifs à suffixe «-age» constituent des cas particuliers: en plus de signifier le fait de faire quelque chose, ils peuvent désigner un ensemble d'éléments, rejoignant ainsi le double-sens des noms verbaux anglais en «-ing». Par exemple, le voligeage représente l'ensemble des voliges et non uniquement le fait de fixer les voliges de la même façon que le terme anglais roofing désigne à la fois le toit et l'activité consistant à le construire.

\section{CONCLUSION}

Malgré la difficulté de travailler dans un groupe si important, nous avons pu, grâce à ce travail, saisir la réalité d'une recherche terminologique dans son ensemble et faire l'expérience de la rédaction d'un article. Nous tenons à remercier un certain nombre de personnes qui nous ont aidées dans notre travail et sans l'assistance desquelles nous n'aurions jamais pu aller au bout de ce projet. Ainsi, nous adressons nos sincères remerciements à $\mathbf{M}^{\text {me }}$ Diane Lacombes, $\mathbf{M}^{\text {me }}$ Nicole Côté et $M$. Pierre Clément, de la CCQ, à M. Serge Thétrault, de l'École des métiers de la construction de Montréal et à M. André Clas, bien sûr, notre professeur, qui nous a guidées tout au long de cette expérience.

\section{Notes}

1. L'anglais distingue stone masonry et brick masonry, selon le matériau utilisé, tandis que le français fait une distinction entre maçonnerie structurelle et maçonnerie de finition, basée sur la partie de la construction exécutée.

2. M. Serge Tétreault, enseignant en briquetage-maçonnerie à l'École des métiers de la construction de Montréal.

\section{Cécile Bruneau, Marina de Almeida, Margreet de Rooij, Marie-HÉlène}

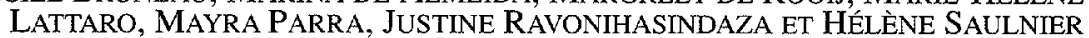

\section{RÉFÉRENCES}

ACI 116R-85 (1985): Cement and Concrete Terminology, 3rd ed., Reported by ACI Committee 116, Publication SP-19 (85) (American Concrete Institute), 58 p.

ADAMS, J. T. (1979): The Complete Concrete, Masonry and Brick Handbook, New York, Arco, 1113 p. ALTH, Max (1978): Masonry and Concrete Work, New York, Harper \& Row.

ANTOINE, F. (1994): Vocabulaire des engins et matériels de chantier, Paris, La Maison du dictionnaire, iv $+61 \mathrm{p}$. Association canadienne des constructeurs (1994): Guide du constructeur, Ottawa, ACCH, $337 \mathrm{p}$.

Banque de terminologie du Québec (1982): États terminologiques et bibliographies, _ Construction, Québec, Editeur officiel du Québec, 247 p.

BARBIER, Maurice (1988): Dictionnaire du bâtiment et des travaux publics, $10^{\mathrm{e}}$ édition, Paris, Eyrolles, $171 \mathrm{p}$.

BÉAL, Christine (1993): Masonry Design and Detailing. For Architects, Engineers, and Contractors, 3rd edition, New York, McGraw-Hill Inc., xix +537 p.

BEAUDRY, Michel (1988) : La charpente en bois, Québec, Les Publications du Québec, $107 \mathrm{p}$.

BELLE-ISLE, J.-Gérald (1977): Dictionnaire technique général, $2^{2}$ éd., Québec, Éd. Beauchemin, 553 p.

BERKOWICZ, Michel (1990): La chaux pour bâtir et décorer, coll. «Nathan Pratique», Paris, 73 p.

BIANCHINA, Paul (1986): Illustrated Dictionary of Building Materials and Techniques, 1 st edition, Blue Ridge Summit, PA, TAB Books Inc., viii +243 p.

BOIVIN, Gilles (1992): Lexique du bâtiment et de quelques autres domaines apparentés, $2^{\mathrm{C}}$ éd., France Michel, Québec, Publications du Québec, 49 p.

BRIGAUX, Guy et coll. (1981): La maçonnerie, $12^{\mathrm{e}}$ ed., Paris, Eyrolles, xv +534 p.

BROCHEN, Alix et coll. (1995): Les mots de la maison, vocabulaire français, anglais et allemand de l'acquisition, de la construction et de la restauration, coll. «Guide House Book», Paris, Éd. Eyrolles, 339 p. 
BROOKS, Hugh (1976): Illustrated Encyclopedic Dictionary of Building and Construction Terms, Englewood Cliffs, NJ, Prentice-Hall, 366 p.

CAGNACCI SCHWICKER, Angelo (1972): International Dictionary of Building Construction, (ang. fr. all. it.), Paris, Dunod, $1261 \mathrm{p}$.

CANON, Kenneth et F. G. HARTLEY (1984) : Technologie de la construction de bâtiments, Montréal, McGraw-Hill, $329 \mathrm{p}$.

CHING, Francis D. K. (1975) : Building Construction Illustrated, New York, Van Nostrand Reinhold, 310 p.

Commission de la Construction du Québec (1991): Les métiers de l'industrie de la construction, $28 \mathrm{p}$.

Conseil de la Construction du Québec (s.d.) : Lexique pratique en briquetage-maçonnerie.

Conseil international de la langue française (1976): Vocabulaire du béton, Conseil international de la langue française, Paris, Eyrolles, $191 \mathrm{p}$.

CORBEIL, Jean-Claude et A. ARCHAMBAULT (1994): Le Visuel multilingue, Montréal, Éd. Québec/Amérique, $\mathrm{xxx}+959 \mathrm{p}$.

CYR, Benjamin (1973): Élêments de construction appliqués à l'habitation, Service des cours par correspondance, Québec, ministère de l'Éducation, $329 \mathrm{p}$.

DALZELL, J. Ralph et G. TOWNSEND (1973): Masonry Simplified, vol. 1, 3rd ed., Chicago, American Technical Society, $408 \mathrm{p}$.

Dictionary of Occupational Titles (1977): 4th ed., U.S. Department of Labor Employment and Training Administration, $\mathrm{xli}+1371 \mathrm{p}$.

Dictionnaire des termes et expressions de l'industrie du béton et de la construction (ang/fr) (1970): Les Éditions Sola, Alouette, Canada, iv + (pag. multiple).

DRYSDALE, Robert G. et coll.(1994): Masonry Structures : Behavior and Design, New Jersey, Prentice Hall, xxvi $+784 \mathrm{p}$.

DUBUISSON, Bemard (1959): Encyclopédie pratique de la construction et du bâtiment, 2 tomes, Paris, Quillet.

DUSSERE-TELMON, Pierre (1988): Dictionnaire vivant du bâtiment et des travaux publics, Quissac, Éd. Dusserre-Telmon, $181+7 \mathrm{p}$.

DUTHU, Henri (1989): Nouvelle encyclopédie de la construction, 2 vol., $3^{\mathrm{e}}$ éd. revue et augmentée, Paris, Éd. du Moniteur, lxxiii $+886 \mathrm{p}$.

Éditions Grammont (1981): Bricolage, l'encyclopédie pour tout faire soi-même à la maison, 9 vol., Lausanne, Grammont.

Éditions Time-Life (1985) : Portes et fenêtres, L'encyclopédie Time-Life du bricolage, Amsterdam, Time-Life, $128 \mathrm{p}$.

FIGOLI, Yves (1983-1988) : L'art de bâtir, 9 vol., Québec, Modulo Éditeur.

FORBES, J. R. (1988) : Dictionnaire d'architecture et de construction français-anglais et anglais-français, $2^{\mathrm{e}}$ éd. Paris, Lavoisier, xii + $416 \mathrm{p}$.

FORBES J. R. (1995): Dictionnaire d'architecture et de construction - Dictionary of architecture and construction, $3^{\mathrm{e}}$ éd. revue et augmentée, Technique et documentation Lavoisier, Paris, Secaucus, NJ, $439 \mathrm{p}$.

FRANE, James T. (1994): Encyclopedia of Construction Terms, Carlsbad, CA, Craftsman Book Company.

GASC, Y. et R. DELPORTE (1979): Les charpentes en bois, coll. «Traité du bâtiment», Paris, Éd. Eyrolles, 323 p.

GAUZIN-MGLLER, Dominique (1990): Le Bois dans la construction, Paris, Éd. du Moniteur, 382 p.

GOLDIN, Georges (1995) : Structure en bois, $4^{\mathrm{e}}$ éd., Montréal, édition de l'auteur, iv + pages multiple.

GOSSELIN, Robert (1995): Lexique anglais-français/français-anglais sur le béton, ses composantes et les nouvelles technologies reliées à sa fabrication, $2^{\mathrm{e}}$ édition - oct. 1995, Rédaction à la page, Montréal, $\mathrm{v}+282 \mathrm{p}$.

Gouvernement du Québec (1989): Les portes et les fenêtres, Québec, ministère de l'Énergie et des Ressources, Bureau de l'efficacité énergétique, $24 \mathrm{p}$.

Grand Larousse en cinq volumes (1987): Paris, Larousse, 5 vol.

Grand Larousse Universel (1989): Dernière édition revue et corrigée, Paris, Larousse, 15 vol.

GROSS, James G. et H. C. PLUMMER (1970): Principles of Clay Masonry Construction, Virginia, Structural Clay Products Institute, $69 \mathrm{p}$.

HARRIS, Cyril M. (Ed.) (1975): Dictionary of Architecture and Construction, New York, McGraw-Hill, xv + 553 p.

ISO (1984): Bâtiment et génie civil - Vocabulaire général - Partie l, ISO 6707/1-1984 (F), première édition, $1984-04-15,25 \mathrm{p}$.

JALIL, Wolfgang A. et coll. (1987): Lexique anglais français \& français anglais des termes du bâtiment et des travaux publics (gros cuvre), Paris, Eyrolles, $\mathrm{xi}+205 \mathrm{p}$.

KREH, R. T. Sr. (1978): Advanced Masonry Skills, Albany, NY, Delmar, vii + 480 p.

Le Nouveau Petit Robert. Dictionnaire alphabétique et analogique de la langue française (1993): nouvelle édition remaniée et amplifiée sous la direction de Josette Rey-Debove et Alain Rey, DICOROBERT Inc., Montréal.

LEFEBVRE, Marcel (1965): Dictionnaire du bâtiment, Building Terms Dictionary, Montréal, SCHL et la Division des recherches en bâtiment du Conseil national de recherches, Leméac, $356 \mathrm{p}$. 
LEFEBVRE, Marcel (1971): (Nouveau) dictionnaire du battiment - Building terms dictionary (new), Montréal, Leméac, $411+46 \mathrm{p}$.

LESSARD, Michel et H. MARQUIS (1972): Encyclopédie de la maison québécoise, Montréal, Les Éd. de l'Homme, $727 \mathrm{p}$.

LONDON, Mark et D. BUMBARU (1984): Fenêtres traditionnelles, Guide technique n² 2, Montréal, Héritage Montréal, $64 \mathrm{p}$.

MACLEAN, James (1989): Elsevier's Dictionary of Building Construction - French-English / English-French, Amsterdam, New York, Elsevier, $8+389 \mathrm{p}$.

MAGUIRE, Byron W. (1978): Masonry and Concrete, Reston, Virginia, Reston Publishing Company, $x+299$ p.

MARSH, Paul. (1982): Illustrated Dictionary of Building, London, New York, Construction Press, $256 \mathrm{p}$.

MATANA, Michel (1989) : Maçonnerie I: terrassements, béton armé, fondations, assainissement, coll. «Concevoir et construire», vol. 1, Paris, Syros-Alternatives, $125 \mathrm{p}$.

MATANA, Michel (1989): Maçonnerie II : mortiers, murs, isolation, cloisons, coll. «Concevoir et construire», vol. 2, Paris, Syros-Alternatives, $125 \mathrm{p}$.

MATANA, Michel (1989) : Maçonnerie III : planchers, carrelages, cheminées, enduit, coll. «Concevoir et construire», vol. 3, Paris, Syros-Alternatives, $125 \mathrm{p}$.

MATANA, Michel (1990): Charpentes : ossature bois, planchers, assemblages, lucarnes, escaliers, coll. «Concevoir et construire», Paris, Syros-Alternatives, $125 \mathrm{p}$.

MCKAY, W. B. (1973) : Brickwork, London, Longman, 196 p.

Ministère de l'Éducation du Québec (1986): Les couvertures, Québec, Publication du Québec, $117 \mathrm{p}$.

Ministère de l'Éducation du Québec (1991): Guide d'organisation pédagogique et matérielle, Construction, briquetage-maçonnerie, Québec, Publication du Québec.

Ministère de l'Éducation du Québec (1991): Programme d'études. Construction, briquetage-maçonnerie, Québec, Publication du Québec.

Ministère des Approvisionnements et Services du Canada (1993): Série d'analyses des professions. Briqueteurmaçon / briqueteuse-maçonne, Québec, Publication du Québec, 145 p.

MONTAGUE, Don (1996): Dictionnaire du bâtiment et du Génie Civil/Dictionary of Building and Civil Engineering, London, Spon.

MORRIS, Christopher (Ed.) (1992): Academic Press Dictionary of Science and Technology, San Diego, Toronto, Academic Press, xxxii $+2432+24$ p.

Office de la langue française (1983): Vocabulaire général de l'outillage (anglais-français), Québec, Office de la langue française, $75 \mathrm{p}$.

Office de la langue française (1990): Glossary of bricklaying/Lexique de briquetage, Québec, Office de la langue française.

OTUA (Office Technique pour l'Utilisation de l'Acier) (1992): Lexique de construction métallique et de résistance des matériaux, Paris, CPS Publications, $121 \mathrm{p}$.

PARÉ, Gilbert (1993): Vocabulaire de la maçonnerie, avec lexique anglais-français, Montréal, Les Éditions de l'Équerre, $83 \mathrm{p}$.

Presses polytechniques et universitaires (1991): Guide de la Technique II - les matériaux, tome 2, Presses polytechniques et universitaires romandes, Lausanne, $x+229 p$.

QUILLET (1968) : Encyclopédie du bâtiment, 5 tomes en 6 vol., Paris, Éd. Eyrolles.

RANDALL, Frank A. et W. C. PANARESE (1976): Concrete Masonry Handbook: For Architects, Engineers, Builders, Illinois, Portland Cement Association, $\mathrm{xv}+211 \mathrm{p}$.

SAUVAGEAU, Jean (1970): Dictionnaire des termes et expressions de l'industrie du béton et de la construction, 2 vol., Québec, Les Éd. Sola Enr.

SCOTT, S. John (1984) : Dictionary of Building, 3rd edition, New York, Halsted Press, $382 \mathrm{p}$.

Sélection du Reader's Digest (1982): Manuel complet du bricolage, $2^{\mathrm{e}}$ édition, Montréal, Sélection du Reader's Digest, $600 \mathrm{p}$.

Sélection du Reader's Digest (1994) : Manuel complet du bricolage, Montréal, Sélection du Reader's Digest, $528 \mathrm{p}$.

SMIT, Kornelis et H. CHANDLER (Ed.) (1991): Means Illustrated Construction Dictionary, Kingston MA, Means, $691 \mathrm{p}$.

Sterling Publishing (1980): The Complete Book of Masonry, Cement \& Brickwork, New York NJ, Sterling Publishing, $154 \mathrm{p}$.

The New Encyclopaedia Britannica (1974): Volume 15, Building Construction, pp. 322-349.

The Portland Cement Association (1988): The Homeowner's Guide to Building with Concrete, Brick \& Stone, Emmaus, PA, Rodale Press, vii +231 p.

TURAUD, Jean (1988): La construction des bâtiments, Technologie de gros auvre et de second œuvre, $4^{\mathrm{e}}$ éd. Paris, Éd. du Moniteur, 348 p.

VIGAN, Jean de (1990) : Dicobat, Dictionnaire général du bâtiment, Paris, Éd. Arcature, $957 \mathrm{p}$ 
VIGAN, Jean de (1992) : Dicobat : dictionnaire général du bâtiment, avec la collaboration du C.S.T.B. pour la correction et la révision des principales définitions, Ris-Orangis, Éd. Arcature, $957 \mathrm{p}$.

VIGAN, Jean de (1993): Dicobat, Dictionnaire général du bâtiment, Paris, Éd. Arcature, $1114 \mathrm{p}$.

WAUGH, R. Hebert et coll. (1954): Handbook of Building Terms and Definitions, New York, Simmons-Boardman Publishing Corporations, ix $+421 \mathrm{p}$.

\section{ANNEXE 1}

Fiches terminologiques

acier $m$ steel

«Alliage métallique de fer et de carbone (.) présentant des caractéristiques très diverses selon le mode de fabrication et de traitement mécanique et thermique (.), et selon les éléments qui lui sont (.) incorporés (alliages).»

Obs.: L'acier est un composant essentiel de la construction, qui sert dans les structures autoporteuses (poutrelles, pieux, charpentes, etc.), les armatures du béton armé, les habillages (tôles, bardage, etc.) et le matériel de chantier.

VIGAN92

Matériaux

CBR

additif m

Voir adjuvant

adjuvant, additif $\mathrm{m}$ admixture, additive

«Produit qui, incorporé à faible dose à un béton, un mortier ou un coulis au moment de leur malaxage, ou étendu à la surface d'un béton ou d'un mortier lorsqu'ils sont encore frais, provoque les modifications recherchées de leurs propriétés habituelles.»

Obs.: On distingue, en fonction des propriétés modifiées, les plastifiants, les fluidifiants ou réducteurs d'eau, les entraîneurs d'air, les accélérateurs ou retardateurs, les hydrofuges, les antigels et les antigélifs.

CILF76:11

Matériaux

CBR

aiguille, aiguille pendante $f$ tie, brace

Poutre verticale, ou parfois oblique, d'une ferme complexe, portant une seule panne et ne travaillant qu'en traction, en principe, pour soutenir l'entrait.

Toiture

MHL

aiguille pendante $f$

Voir aiguille

aisselier $m$ brace

Poutre oblique d'une ferme, travaillant en compression et en traction, ayant une fonction de consolidation car il maintient un angle entre deux poutres.

Obs. : Voir observation à compression

Toiture

MHL

allège $\mathrm{f}$ apron wall, breast, window breast, breast wall Partie du mur comprise entre le sol et la tablette d'appui d'une fenêtre, ayant la même largeur que la baie. Ouvertures

HS alliage $\mathrm{m}$ alloy

Produit métallurgique obtenu par l'incorporation à un métal d'un ou de plusieurs éléments, métalliques ou non. Obs.: L'alliage permet de modifier certaines des propriétés d'un métal ou de lui en conférer de nouvelles. Dans le bâtiment, on utilise de nombreux alliages réalisés surtout à base de cuivre et d'aluminium. On dit «allier un métal à /avec/et un autre». On dit «réaliser un alliage».

Matériaux

MDA

aluminium $m$ aluminum

Métal léger, ductile et malléable, peu oxydable à l'air, pouvant avoir de nombreuses applications dans la construction, soit pur, soit sous forme d'alliages.

Obs. : L'aluminium peut être travaillé par laminage, filage, étirage, emboutissage, estampage et forgeage. Il peut être assemblé par soudage, collage, rivetage, vissage ou clouage. Il est utilisé pour faire des profilés d'huisserie, des joints, des tôles, des bacs de couverture, des bardages de façade, des charpentes légères, etc. Matériaux

CBR

aplainissoir $\mathrm{m}$ float

Règle dotée d'une poignée, employée par le plâtrier, qui sert à lisser le ciment.

Maçonnerie

MSP/MDR

appui $m$ tablette d'appui f sill, window sill, outer sill «Partie inférieure d'une baie sur laquelle repose la croisée [qui] désigne, en maçonnerie, la tablette de couronnement d'une allège, en légère pente pour l'écoulement des eaux vers l'extérieur.»

Obs. : Ne pas confondre avec traverse d'appui. L'appui, ou tablette d'appui, fait référence à la baie, alors que la pièce d'appui, ou traverse d'appui, fait référence au bâti dormant de la fenêtre.

VIGAN92:73

Ouyertures

HS

arbalétrier $\mathrm{m}$ membrure supérieure f principal rafter, sloping beam, top chord, top member

Poutre principale d'une ferme qui, prenant généralement appui sur l'extrémité de l'entrait et sur le sommet du poinçon, soutient les pannes intermédiaires. Toiture

MHL

ardoise $f$ slate

Roche schisteuse qui a pour particularité de se débiter en feuillets minces, surtout utilisée pour la couverture des maisons. 
Obs. : L'ardoise sert aussi au revêtement des sols, des terrasses, des marches, aux revêtements muraux, etc. Matériaux

MDA

arêtier $\mathrm{m}$ hip

«Ligne inclinée de rencontre de deux versants d'une toiture inclinée formant un angle saillant.»

Obs. : La contrepartie est noue.

ISO6707/1:10

Toiture

MHL

argile, glaise f clay

Roche terreuse sédimentaire compacte, imperméable et meuble, constituée essentiellement de silicate d'aluminium et de fer et entrant comme matière première dans la fabrication des ciments et des produits céramiques comme les briques, tuiles, carrelages, céramiques, claustres, boisseaux, etc.

Obs. : On retrouve l'adjectif argileux.

Matériaux

CBR

asphalte $m$ asphalt

Composition rocheuse brun très foncé de schiste, de calcaire ou de silice, contenant au moins $6 \%$ de bitume naturel.

Obs.: Pour qu'il forme le mastic d'asphalte, on enrichit souvent l'asphalte de bitume, jusqu'à $11 \%$ ou même $17 \%$.

Toiture

JRS

baie $f$ opening, aperture

«Toute ouverture pratiquée dans un mur ou une toiture, ayant pour objet le passage ou l'éclairage des locaux.» Obs.: Cette ouverture peut être vide ou fermée par une porte ou une fenêtre. On dit baie de porte ou baie de fenêtre.

VIGAN92

Ouvertures

HS

baie vitrée $f$

Voir fenêtre panoramique.

bardeau, tavaillon $\mathrm{m}$ shingle

Petite planche de 30 à $50 \mathrm{~cm}$ de largeur destinée à couvrir des bâtiments rustiques.

Obs.: Le bardeau redevient à la mode, soit comme bardeau-bois qui est un matériau très cher, soit comme bardeau bitumé et mesure un à deux mètres. Toiture

JRS

bardeau bitumé $m$ bitumen shingle

Matériau en général composé d'une ou de deux armatures de fibres de verre mêlées d'amiante et enrobées de bitume.
Obs. : La couche de divers granulés constituant sa surface extérieure offre une grande variété de teintes. Sa pose peut se faire soit «à l'américaine», lorsqu'on le cloue sur un support continu, soit «à la française», lorsque des crochets le fixent sur des lattes de bois, de la même façon que des couvertures d'ardoises.

Toiture JRS

\section{barre inclinée $\mathrm{f}$}

Voir diagonale

\section{barre verticale $f$}

Voir montant

bâti dormant, dormant $m$, huisserie $f$ window frame Encadrement fixe dans une baie, généralement à feuillures, scellé à la maçonnerie et destiné à recevoir une partie ouvrante telle que porte, fenêtre, etc.

Obs. : Le bâti dormant est composé de deux éléments verticaux appelés montants, d'un élément horizontal supérieur appelé traverse, et d'un élément horizontal inférieur appelé pièce d'appui. Le terme est employé par opposition à ouvrant, partie mobile de la fenêtre. Le terme huisserie désignait, à l'origine, le bâti dormant de la porte. Par extension, il est employé pour désigner la partie dormante de toute baie.

Ouvertures

HS

battant, vantail $m$ casement, window casement Châssis mobile d'une fenêtre s'ouvrant par rotation autour d'un axe vertical ou horizontal.

Obs. : À l'origine, vantail et battant désignaient les parties mobiles des fenêtres ouvrant à la française. Ils sont aujourd'hui employés pour désigner tout genre de châssis mobile s'ouvrant par rotation.

Ouvertures

HS

bédane $\mathrm{f}$ vide joints $\mathrm{m}$ mortise chisel

Ciseau en pointe, avec une rainure à l'intérieur, qui sert à dégrader, à ravaler les joints et à enlever les éléments de maçonnerie.

Obs. : En anglais britannique on écrit mortice chisel.

Maconnerie

MSP/MDR

béton $m$ concrete

Matériau de construction obtenu par agrégation de granulats (sable, gravier et cailloux) au moyen d'un liant (ciment, chaux), d'eau et, éventuellement, d'un adjuvant.

Obs. : Le béton permet de réaliser par moulage, banchage, projection ou coffrage, toutes sortes de pièces et de volumes qui, après durcissement à l'air, présentent une bonne cohésion et une résistance élevée, surtout en compression.

Il existe une centaine de variétés de ciments.

Matériaux

CBR 
béton armé $m$ reinforced concrete

Association de béton et d'acier pour obtenir un matériau présentant une bonne résistance aux diverses sollicitations.

Obs. : Le béton armé est utilisé dans les fondations, les planchers, les murs, les poteaux, l'ossature, etc., soit les éléments porteurs d'une construction. Le béton armé aurait tendance à être supplanté par un autre type de béton, le béton précontraint. Bien que plus performant, ce dernier est beaucoup moins souple, étant donné qu'il est pratiquement impossible de le recouper et de le restaurer, comme c'est le cas pour le béton armé.

Matériaux

MDA

béton d'argile $\mathrm{m}$ rammed clay

Mélange de granulats et d'argile, stabilisé par de la chaux ou du ciment.

Obs.: Lorsque l'on utilise de la terre argileuse contenant naturellement les granulats nécessaires pour réaliser ce béton, il est alors appelé béton de terre. Matériaux

\section{MDA}

béton gras $m$ fat concrete

Béton ayant un taux élevé de liant et de charges fines, ce qui permet un enrobage complet des granulats.

Obs.: Plus l'enrobage des granulats est complet, plus les frottements sont réduits lors de la mise en œuvre. Ainsi, contrairement à un béton maigre, un béton gras présente une plus grande ouvrabilité, soit l'aptitude d'un béton ou d'un mortier à être mis en œuvre ou travaillé.

Matériaux

MDA

béton maigre $\mathrm{m}$ lean concrete

Béton ayant un taux peu élevé de liant et de charges fines, ce qui ne permet qu'un enrobage partiel des granulats.

Matériaux

MDA

bétonnière $f$ concrete mixer, cement mixer

Machine à cuve rotative, munie de pales fixes, qui sert à malaxer mécaniquement les composants des enduits.

Maçonnerie

MSP/MDR

béton précontraint $\mathrm{m}$ prestressed concrete

«Béton (.) soumis, avant sa mise en service, à un effort de compression permanent visant à empêcher l'apparition, en service, de contraintes de traction.»

Obs.: La mise en précontrainte s'effectue avant le bétonnage, par appui sur le béton durci (post tension), ou après, par fils adhérents tendus (prétension).

VIGAN92

Matériaux

MDA

\section{biellette $f$ lever}

Petite tige rigide, articulée aux deux extrémités et destinée à la transmission du mouvement entre deux pièces mobiles.

Ouvertures

HS

bitume $\mathrm{m}$ bitumen

Mélange d'asphalte et de résidus du pétrole brut, entièrement dissous par le sulfure de carbone.

Obs. : Le bitume est le plus dense des produits obtenus par distillation du pétrole. Très adhésif, lorsqu'il est liquéfié à chaud, il sert aussi bien au revêtement routier qu'à tous les produits d'étanchéité. On trouve le bitume naturel, contenant $20 \%$ de calcaire et le bitume de pétrole qui, obtenu par distillation du pétrole, est lourd et très peu volatil.

Toiture

JRS

bloc de ligne $m$

Voir coin.

blochet $m$ tie beam piece, tie brace

Petite poutre horizontale qui solidarise, dans les fermes à entrait retroussé, la jambe de force à la panne sablière ou au pied de l'albalétrier, contribuant à l'invariabilité de l'angle formé par la jambe de force et l'entrait.

Obs.: Voir les observations à compression.

Toiture

MHL

bois $\mathrm{m}$ wood

Matière vivante constituant l'ossature des végétaux. Désigne la partie massive des troncs ou fûts des arbres utilisables comme matériau de construction et les ouvrages en bois.

Obs.: On distingue sur un tronc d'arbre plusieurs zones distinctes qui sont l'écorce, l'aubier, le duramen et la mœlle. Le bois est caractérisé par plusieurs facteurs physiques : dureté, densité, retrait/rétractabilité, taux d'humidité, etc. Il existe différents types de sections du bois : madriers, bastaings, chevrons, demichevrons, planches d'échafaudage, planches lorraines, lambourdes, planchettes /feuillets, frises, voliges, demivoliges, lattes, tasseaux, etc. On retrouve aussi en anglais les équivalents lumber et timber. Selon certains auteurs, ils auraient tous deux des sens plus spécifiques: lumber représenterait le bois de construction/ bois en grumes, mais aussi toute pièce de bois; timber serait le bois d'œuvre/bois de charpente, mais aussi le bois de construction. Ainsi, il apparaît que les notions se recouvrent Matériaux

CBR

boîte à mélange, boîte de gâchage, panne à mortier $f$ mortar box, mortar pan

Récipient rectangulaire en bois, en plastique ou en tôle, aux bords évasés, qui sert à préparer ou à gâcher un enduit.

Maçonnerie

MSP/MDR 
boîte de gâchage $\mathrm{f}$

Voir boîte à mélange.

boucharde $f$ bush hammer

Outil de frappe, muni d'une tête carrée, hérissée de dents pyramidales en acier, qui sert à obtenir une finition particulière sur une surface en ciment ou en mortier frais, ou à frapper la surface de la pierre afin d'obtenir une finition particulière.

Obs. : S'écrit aussi en anglais bush-hammer.

Maçonnerie

MSP/MDR

bouclier $\mathrm{m}$ float, laying-on trowel

Outil de maçon ou de plâtrier, avec une semelle de bois ou d'acier, qui sert à porter le mortier ou l'enduit à appliquer ainsi qu'à resserrer ou à damer les enduits de ciment.

Obs.: En anglais, on appelle float l'outil que l'on utilise pour resserrer le plâtre alors que celui que l'on utilise pour appliquer le plâtre est nommé laying-on trowel.

Maçonnerie

MSP/MDR

\section{brique $\mathrm{f}$ brick}

«Matériau céramique préfabriqué, traditionnellement à base d'argile cuite, dont les formes géométriques et calibrées sont obtenues soit par moulage, soit par extrusion (ou filage).»

Obs.: La brique est un composant de base de la construction de parois verticales. On distingue differents types de briques selon leur procédé de fabrication, de leur fonction, de leur forme, de leurs particularités et de la proportion des vides compris dans la brique. Le format des briques est le plus souvent $4 \times 10^{5} \times 22,5^{4} \times 10^{5} \times 22$ ou $6 \times 10^{5} \times 22 \mathrm{~cm}$. Les briquettes, les briquetons, les trois-quartiers, les mulots et les tuileaux sont des briques de dimensions inférieures. On dit «poser des briques», «graisser la brique». PARE93: 17 + VIGAN92

Matériaux

CBR

brique à angle $\mathrm{f}$ angle brick

Brique dont la forme permet de réaliser des murs à angle obtus.

Matériaux

CBR

brique claveau, brique en coin $\mathrm{f}$ arch brick, compass brick, voussoir

«Brique présentant des faces non parallèles, permettant l'exécution de cheminées, d'arcs, de voûtes, etc.»

Obs.: Il existe de nombreux synonymes en anglais comme en français. Le choix des termes s'est donc fait en fonction de leur frequence d'utilisation. PARE93

Matériaux

CBR brique commune $\mathrm{f}$

Voir brique ordinaire

brique creuse $f$ air brick

Brique parallélépipède dont l'ensemble des perforations a une section cumulée supérieure à $40 \%$ de la section de l'élément et qui est utilisée dans la maçonnerie armée ou pour le doublage.

Obs. : On distingue, par exemple, dans cette catégorie les briques creuses courantes, les briques plâtrières, les briques à rupture de joint, les briques isolantes, etc. Matériaux CBR

brique de parement $\mathrm{f}$ facing brick, face brick «Plaquette de terre cuite, destinée à la décoration de murs intérieurs ou extérieurs.»

BROCHEN95

Matériaux

CBR

brique de pavage $f$ paving brick

«Brique offrant une résistance élevée à l'abrasion et au gel-dégel, utilisée à l'extérieur comme revêtement de sol.»

PARE93: 17

Matériaux

CBR

\section{brique en coin $\mathrm{f}$}

Voir brique claveau

brique extrudée $f$ wirecut brick

«Brique fabriquée par extrusion et coupage, avec de l'argile contenant relativement peu d'eau.»

Obs: S'écrit aussi en anglais wire-cut brick.

PARE93

Matériaux

CBR

brique ordinaire, brique commune $\mathrm{f}$ building brick, common brick

Brique parallélépipède rectangle à faces planes, communément employée en construction et non traitée pour la texture ou la couleur, pouvant être brûlée ou vitrifiée.

Matériaux

CBR

brique perforée f perforated brick, cored brick

«Brique traversée de perforations perpendiculaires aux lits de pose, dont la somme des sections des trous est inférieure ou égale à $\mathbf{4 0} \%$ de la surface du lit de la brique.)

VIGAN92

Matériaux

CBR

brique pleine $f$ solid brick

Brique parallélépipède rectangle à faces planes ne comportant aucun vide

Matériaux

CBR 
brique réfractaire $\mathrm{f}$ fire brick

Brique résistant à de très fortes températures et que l'on utilise pour les endroits soumis à de hautes températures (foyers, poêles, barbecues, etc.).

Obs: S'ecrit aussi en anglais firebrick.

Matériaux

CBR

brique sablée f sand-faced brick

«Brique moulée dont les faces sont recouvertes de sable.»

PARE93: 16

Matériaux

CBR

briqueteuse $\mathbf{f}$

Voir truelle briqueteuse.

calcaire $\mathrm{m}$ limestone

Ensemble des roches sédimentaires constituées de carbonate de calcium $\left(\mathrm{CaCO}_{3}\right)$, matière première dans la fabrication des chaux et des ciments, et utilisées comme pierres de construction ou comme granulat.

Obs.: Le calcaire est aussi appelé pierre à chaux. L'origine des roches calcaires est organique (calcaires organogènes, craies, tuffeaux, calcaire tendre, entroque, coquiller), chimique (tuf, travertins, calcaires oolithiques) ou détritique (marbres et brèches calcaires). Matériaux

CBR

cale $f$ wedge

Pièce de bois taillée en biseau qui sert à immobiliser un élément quelconque en maçonnerie.

Maçonnerie

MSP/MDR

carreau m

Voir vitre.

céramique $f$ ceramic, ceramics

Dans le bâtiment, le travail de l'argile qui, mélangée avec de l'eau, devient dure et inaltérable après cuisson, ainsi que les matériaux résultant de ce travail. Obs. : Les auteurs anglais ne semblent pas s'accorder sur l'aire sémantique recouverte par le terme ceramics. Pour certains, ceramics désigne l'art céramique alors que pour d'autres, ce terme se réfere aux matériaux. Parmi les céramiques traditionnelles, on distingue principalement les céramiques poreuses et les céramiques imperméables. Les produits céramiques poreux ont une cassure terreuse et une pâte perméable, et ils comprennent, d'une part, les produits de terre cuite plus ou moins colorée en rouge (tuiles, briques, conduites de fumée, carreaux de dallage, etc.) et, d'autre part, les poteries à pâte blanche et fine, cuites à plus haute température que les terres cuites et recouvertes d'un émail transparent, les faïences (vaisselle de table, pièces décoratives, carreaux de revêtement, etc.). Quant aux produits céramiques imperméables, dont la pâte a été vitrifiée à haute température, ils comprennent les grès cérames dont la pâte est dure et pratiquement opaque (carreaux de dallage, tuyaux, etc.), les porcelaines (vaisselle, pièces à usage technique et de laboratoire, matériel électrique, etc.), et les céramiques sanitaires.

Matériaux

MDA

chambranle $\mathrm{m}$ casing, architrave

«Encadrement rapporté en applique au pourtour de l'huisserie d'une fenêtre; se compose de deux montants verticaux réunis à leur sommet par une traverse horizontale.*

Obs. : S'emploie davantage avec porte qu'avec fenêtre. VIGAN92

Ouvertures

HS

chanlatte $f$ verge board

Latte de bois au pied des chevrons de charpente, recevant la première rangée de tuiles ou d'ardoises.

Obs.: Lorsque la chanlatte est plus large et moins épaisse, on l'appelle volige chanlattée et elle sert alors de voligeage.

Toiture

JRS

charpente (de toit) $f$ (roof) framing, frame, framework Ensemble des pièces composant l'ossature de la toiture conçu pour résister aux charges et aux efforts dus à la neige, au vent, et autres, et qui assure la cohésion et la rigidité de la toiture.

Toiture

MHL

châssis m casement, window casement, sash

«Composant, fixe ou ouvrant, constitué essentiellement d'un cadre recevant une partie vitrée, grillagée, pleine ou à lamelles.»

Obs.: Le châssis fixe est aussi appelé châssis dormant, par opposition à châssis ouvrant, ou ouvrant. En anglais: fixed sash, fixed light, deadlight. Châssis est un terme générique qui peut désigner aussi bien le battant de la fenêtre à la française que le châssis de la fenêtre à guillotine. Dans ce dernier cas, l'anglais préfère sash. Au Québec, les détaillants de fenêtres donnent le nom de volet au châssis. C'est un emploi inhabituel car volet désigne un dispositif de fermeture de fenêtre, généralement plein. On remarque toutefois que volet, tout comme battant et vantail, porte le sens de battre au vent.

ISO 6707/1-1984

Ouvertures

HS

châssis à guillotine $\mathrm{m}$

Voir fenêtre à guillotine.

châssis à la canadienne $\mathrm{m}$

Voir fenêtre à la canadienne. 
châssis à la française $m$

Voir fenêtre à la française.

châssis à l'anglaise m

Voir fenêtre à l'anglaise.

châssis à l'australienne $\mathrm{m}$

Voir fenêtre à l'australienne.

châssis à l'italienne m

Voir fenêtre à l' italienne.

châssis basculant m

Voir fenêtre basculante.

châssis coulissant $m$

Voir fenêtre coulissante.

châssis de toiture $m$

Voir fenêtre en toiture.

châssis inférieur $m$ lower sash

Partie fixe ou mobile inférieure de la fenêtre à guillotine. Ouvertures

HS

\section{châssis pivotant $\mathrm{m}$}

Voir fenêtre pivotante.

châssis supérieur $\mathrm{m}$ upper sash

Partie fixe ou mobile supérieure de la fenêtre à guillotine.

Ouvertures

HS

châssis-trémie $\mathrm{m}$

Voir fenêtre à soufflet.

chaume $m$ thatch

Matériau de couverture constitué de tiges de graminées telles que le seigle, de tiges de genêts ou de roseaux.

Obs. : Non seulement très sensible au feu, le toit de chaume exige une main d'œuvre spécialisée et, de ce fait, revient cher. On ne peut l'installer que sur une pente d'au moins $45 \%$, ainsi qu'une charpente capable de supporter une charge minimale de $50 \mathrm{~kg} / \mathrm{m}^{2}$, en plus du poids de la neige et de la glace.

Toiture

JRS

\section{chaux flime}

Liant utilisé en maçonnerie et obtenu par cuisson du calcaire à une très haute température $\left( \pm 1000^{\circ} \mathrm{C}\right)$.

Obs. : On distingue trois types de chaux, soit la chaux vive, sans eau; la chaux aérienne (ou chaux éteinte), utilisée comme mortier ou enduit, résultant du mélange d'eau et de chaux vive et dont la prise s'effectue sous l'action du gaz carbonique de l'air; et la chaux hydraulique, obtenue par cuisson d'un calcaire argileux et dont la prise s'effectue sous l'action de l'eau (par hydratation).

Matériaux

CBR chaux hydraulique f hydraulic lime

Chaux obtenue par la cuisson, à $900^{\circ} \mathrm{C}$ environ, de calcaire contenant naturellement 8 à $20 \%$ d'argile ou d'un mélange équivalent de calcaire et d'argile broyés. Obs.: Cette chaux est dite hydraulique, car elle présente la propriété de prendre par contact avec l'eau. On ajoute de la chaux hydraulique aux mortiers, et à certains bétons, pour améliorer leur plasticité et augmenter ainsi leur ouvrabilité.

Matériaux

MDA

chaux vive f quick lime

Chaux obtenue par décomposition du calcaire lors de sa cuisson à environ $900^{\circ} \mathrm{C}$

Obs. : À la cuisson, le calcaire se décompose en chaux vive $(\mathrm{CaO})$ et en gaz carbonique $\left(\mathrm{CO}_{2}\right)$ (environ $44 \%$ ). La perte importante de gaz carbonique entraîne une forte porosité, ce qui explique la grande réactivité de la chaux vive qui, en présence d'eau (réaction appelée une extinction), donne la chaux aérienne, aussi appelée chaux éteinte ou chaux hydratée. Les chaux aériennes sont dites grasses lorsqu'elles sont obtenues à partir de calcaires très purs; elles sont dites maigres lorsque les calcaires utilisés contiennent des impuretés.

Matériaux

MDA

chevron $m$ rafter

Pièce de bois qui soutient la couverture.

Obs. : Il existe plusieurs sortes de chevrons, comme le grand chevron, common raft, qui est commun à toutes les charpentes de toit en pente et se prolonge de la sablière du mur extérieur au fâttage; le chevron d'angle, hip rafter, qui relie le faitage à l'intersection de deux sablières où il forme une arête à chacun des quatre coins de la maison; le chevron de nove, valley rafter, qui est également un chevron d'angle, mais qui est situé à l'angle droit rentrant formé par deux pentes de couverture; ou le chevron boiteux, criple rafter, qui, sans toucher le faîtage ni la sablière, va d'un arêtier à une noue.

Toiture

JRS

\section{ciment $m$ cement}

«Liant minéral hydraulique en poudre à base de calcaire et d'argile qui, mélangé avec de l'eau, fait prise et permet d'agglomérer entre eux des sables et des granulats pour constituer de véritables roches artificielles, les bétons et les mortiers.»

Obs.: Il existe une cinquantaine de variétés de ciments.

VIGAN92

Matériaux

CBR

ciment à maçonner, ciment à maçonnerie m masonry cement

Mélange de ciment Portland, de calcaire en poudre et d'adjuvants entraineur d'air, donnant un mortier plus souple et réduisant moins qu'un mortier fait de ciment Portland standard.

Matériaux

CBR 


\section{cỉment à maçonnerie $m$}

Voir ciment à maçonner

ciment Portland m Portland cement

Ciment hydraulique très fort, communément utilisé dans le bâtiment, qui contient près de $97 \%$ de clinker avant l'incorporation des produits d'addition.

Obs. : Le ciment Portland est aussi appelé ciment artificiel par opposition au ciment naturel. S'écrit aussi ciment portland.

Matériaux

MDA

ciseau à bouchardage $m$ pitching chisel

Outil muni d'une tête carrée et dentée, qui sert à dégrossir les pierres.

Maçonnerie

MSP/MDR

ciseau à brique, set à brique $\mathrm{m}$ brick set, bolster

Outil muni d'une lame large en biseau et d'une poignée qui sert à couper les briques, à casser la pierre, à buriner le mortier et à enlever les briques brisées.

Maçonnerie

MSP/MDR

ciseau à dents $m$ claw chisel, claw tool, tooth chisel, stonecutter's chisel

Outil muni d'une lame dentée, qui sert à casser un élément de maçonnerie.

Maçonnerie

MSP/MDR

ciseau à froid $m$ chip ax

Outil muni d'une lame coupante, qui sert à casser du béton ou un élément quelconque de maçonnerie.

Maçonnerie

MSP/MDR

ciseau de maçon, ciseau pinch $m$ pointe $f$ raker

Outil muni d'un crochet monté sur un manche, qui sert à gratter ou à dégarnir les joints entre les pierres ou entre les briques.

Maçonnerie

MSP/MDR

ciseau pinch $\mathrm{m}$

Voir ciseau de maçon

clinker m clinker

«Produit obtenu par cuisson jusqu'à fusion partielle (.) d'un mélange dosé et homogénéisé de matières constituées principalement de chaux, de silice, d'alumine et d'oxyde de fer.»

Obs. : Le clinker a des propriétés hydrauliques et c'est le constituant principal du ciment Portland.

CILF76: 58

Matériaux

CBR

coin, bloc de ligne, tendeur à ligne $\mathrm{m}$ comer block, line block, line bobbin
Support en angle, fixé au coin d'un élément de maçonnerie, qui sert à tenir et à tendre la ligne de maçon.

Maçonnerie

MSP/MDR

comble $\mathrm{m}$ roof structure

Ensemble de la construction surmontant un édifice et comprenant la charpente et la couverture.

Obs.: Dans le cas du comble à deux pentes, spanroof, le système de couverture est constitué d'un faîtage et deux rampants, tous deux délimités par une ligne d'égout et de deux rives. C'est, entre autres, le comble à deux pentes qui se construit sur ferme, trussed, parfois avec un avant-toit, umbrella.

\section{Toiture}

JRS

\section{compression $f$ compression}

Contrainte s'exerçant dans le sens longitudinal d'une poutre et provoquant un raccourcissement.

Obs.: En anglais, on nomme stay la pièce qui sert a maintenir la rigidité d'un ensemble, strut celle qui travaille surtout en compression (aisselier, contrefiche, jambe de force et blochet) et brace celle qui travaille en compression (in compression brace: contrefiche, jambe de force) ou en traction (in tension brace : tirant, voir observation à traction).

Toiture

MHL

contre-châssis $m$

Voir contre-fenêtre.

contre-fenêtre, fenêtre de doublage f contre-châssis m storm sash, storm window

Fenêtre disposée en deçà ou au-delà d'une autre pour constituer avec elle une barrière contre le froid ou le bruit.

Obs. : Généralement utilisée avec la fenêtre à guillotine. L'emploi de fenêtre double ou de châssis double est une traduction fautive de double-window. Dans l'usage on tend à confondre contre-fenêtre et doublefenêtre. Plur. : contre-fenêtres.

Ouvertures

$\mathrm{HS}$

contrefiche f strut, stay, brace

Poutre oblique d'une ferme complexe, qui soutient un nœud, et qui est placée entre le nœud qu'elle supporte et la base du poinçon.

Obs.: S'écrit aussi contre-fiche. Voir observation à compression.

Toiture

MHL

contrevent $\mathrm{m}$

Voir volet.

cordeau $\mathrm{m}$

Voir ligne à briqueteur. 
cordeau à craie, cordeau traceur $m$ chalk line

Petite corde couverte de craie, qui sert à marquer une ligne droite entre deux points.

Obs.: La corde est tendue entre les deux points, puis levée et relâchée, afin que la craie laisse une trace sur la surface.

Maçonnerie

MSP/MDR

cordeau d'alignement $\mathrm{m}$

Voir ligne à briqueteur.

cordeau traceur $m$

Voir cordeau à craie.

couteau m knife

Outil de plâtrier, muni d'une lame montée sur un manche, qui sert à appliquer et à niveler un enduit.

Obs.: Il existe différents types de couteaux selon l'uti-

lisation: couteau à platre, couteau à enduire, couteau d'angle, couteau à mastiquer ou à mastic.

Maçonnerie

MSP/MDR

couteau à mastic $m$

Voir couteau à mastiquer

couteau à mastiquer, couteau à mastic m putty knife Outil de plâtrier muni d'une lame large et souple, qui sert à appliquer le mastic.

Maçonnerie

MSP/MDR

couverture f roof-covering

Partie extérieure de la toiture protégeant les structures d'un édifice contre les intempéries telles que la pluie, le vent et la neige, grâce à son étanchéité et sa durabilité.

Obs. : Le matériau de couverture doit être choisi selon des critères précis relatifs à la pente de la toiture, au climat et aux intempéries courants de la région et à la situation de l'édifice.

Toiture

JRS

coyau m sprocket

Petite pièce de bois placée sur la partie inférieure des chevrons pour radoucir la pente d'une couverture.

Toiture

JRS

crépissoir $\mathrm{m}$ brush

Balai dur, à manche court, qui sert à appliquer un enduit sur un mur.

Obs. : On dit crépir un mur.

Maçonnerie

MSP/MDR

croisée $\mathrm{f}$

Voir fenêtre.

croupe f hipped end

Petit versant de toiture délimité dans sa partie inférieure par l'égout et sur ses côtés par deux arêtiers.
Obs. : La croupe peut être triangulaire, polygonale ou arrondie. S'oppose à pignon.

Toiture

JRS/MHL

\section{diagonale $f$ diagonal}

Élément oblique d'une ferme triangulée, qui travaille en traction et en compression.

Toiture

MHL

\section{dormant $m$}

Voir bâti dormant

\section{double fenêtre $f$ double window}

Fenêtre équipée de deux châssis parallèles, un châssis et un contre-châssis ou contre-fenêtre, pour en améliorer les performances acoustiques ou thermiques.

Ouvertures

HS

double vitrage, vitrage isolant, verre isolant $m$ double glazed window, double glazing

Dispositif fabriqué en usine composé de deux vitres emprisonnant une lame d'air déshydratée ou du gaz inerte, ayant pour fonction de renforcer l'isolation.

Obs. : Dans l'usage on tend à confondre double vitrage et survitrage. Le vitrage isolant peut être triple, on parle alors de triple vitrage.

Ouvertures

HS

égout $m$ eaves

Partie basse d'un toit vers laquelle l'eau de pluie coule puis se déverse sur le sol en passant par la gouttière.

Obs. : L'égout est libre, dripping eaves, lorsqu'il n'a pas de gouttière et qu'il laisse l'eau tomber au pied du mur. L'égout est dit droit, lorsqu'il est perpendiculaire à la ligne droite de la grande pente du versant et biais, lorsqu'il forme un angle supérieur à $45^{\circ}$.

Toiture

JRS

embrasure $m$ opening, window opening

Espace vide compris entre les parois du mur dans une baie.

Obs. : Ne pas confondre avec ébrasement qui désigne l'évasement des tableaux intérieurs d'une baie dans le but de donner plus de jour, ou plus de jeu aux battants. Ouvertures

HS

\section{enduit $m$ plaster}

«Couche de plâtre, de mortier, de chaux, de ciment ou de résine, destiné à la protection, à la finition ou à la décoration des parois.»

Obs.: On distingue quatre types d'enduits : les enduits intérieurs (enduit de plâtre, enduit de peintre, enduit de ragréage mural intérieur, enduit de correction acoustique, enduit de jointoiement, enduit de cuvelage, enduit de lissage des sols, etc.), les enduits de façade (ou extérieurs) (enduit d'imperméabilité, enduit 
bicouche, enduit monocouche d'imperméabilisation et de parement, enduit tramé ou roule, enduit de ragréage), les enduits d'étanchéité ou enduits noirs (enduit pâteux, enduit d'application à chaud, enduit d'imprégnation à froid) et les enduits légers ou enduits isolants. CILF76:79

Matériaux

CBR

entrait $m$ tie beam, main tie

Poutre horizontale constituant la base d'une ferme qui empêche les pieds des arbalétriers de s'écarter l'un de l'autre, et qui est soumise à des forces de traction.

Obs. : Voir observations à compression et à traction. Toiture

MHL

entrait retroussé $m$ collar beam, collar tie

Entrait qui n'est pas solidaire du pied des arbalétriers et qui travaille en traction.

Obs.: Voir observations à compression et à traction.

Il est souvent confondu avec le faux-entrait (qui travaille en compression) parce qu'il se trouve à la même position dans la ferme.

Toiture

MHL

équerre de maçon $f$ plumb rule

Instrument triangulaire au bout duquel pend un fil de maçon, qui sert à vérifier l'aplomb de l'assise d'une construction.

Obs.: Le terme anglais désigne un outil dont la forme est différente (une planche rectangulaire), mais la fonction est la même.

Maçonnerie

MSP/MDR

faite $\mathrm{m}$ ridge

Sommet ou partie supérieure d'un ouvrage.

Obs.: Par extension, le faîtage est la pièce horizontale qui forme le sommet de la charpente du toit et sur laquelle repose la tête des chevrons. En plus de permettre le parfait alignement de ces derniers, le faîtage empêche la couverture de se creuser ou de s'affaisser partiellement, si les chevrons viennent à faiblir sous le poids de la neige ou de la glace.

Toiture

JRS

faîtière (1) f ridge-purlin

Couverture spéciale de poterie installee, par glissement ou par emboîtement, par-dessus une ligne de faîte. Toiture

JRS

faîtière $(2) \mathrm{f}$

Voir panne faîtière.

faux-entrait $\mathrm{m}$ false tie beam, horizontal strut between principals

Entrait qui n'est pas solidaire du pied des arbalétriers mais qui est fixé nettement plus haut.
Obs.: Cet entrait est soumis à des efforts de compression, contrairement au véritable entrait, ce qui lui vaut l'épithète de faux. Voir observation à entrait retroussé.

Toiture

MHL

fendeuse $\mathbf{f}$

voir guillotine

fenêtre, croisée $f$ window

Élément de construction, composé d'un bâti dormant et d'un châssis recevant un vitrage, fixé dans une baie verticale ou sensiblement verticale, dans un mur ou une toiture inclinée, qui permet l'éclairage extérieur et éventuellement l'aération.

Obs.: L'usage a consacré l'emploi châssis et fenêtre comme synonymes. Au Québec, en particulier, on dira «ferme la fenêtre» ou «ferme le châssis». On remarque la distinction, dans la définition, entre fenêtre (élément de construction) et baie (ouverture). Une fenetre peut être simple ou composée (combination window); il s'agit alors d'un ensemble de plusieurs châssis fixes ou mobiles, possédant des modes d'ouverture semblables ou différents, et séparés par des parties dormantes. Les fenêtres en saillie (bay window; bowwindow pour fenêtre en arc) sont des fenêtres composées. Croisée est un terme littéraire, peu employé au Québec. En France, on l'emploie comme synonyme de fenêtre, en particulier de fenêtre à battants. Même s'ils sont employés comme synonymes, croisée et fenêtre ont une légère différence de sens: croisée évoque l'idée des montants et traverses, alors que fenêtre évoque davantage l'ouverture. Ainsi on dira «percer une fenêtre» et «fermer la croisée». Ouvertures

HS

fenêtre à battants

Voir fenêtre à l'anglaise

fenêtre à guillotine $f$ châssis à guillotine $m$ sash window, hung window

Fenêtre composée de châssis coulissant verticalement les uns contre les autres. Le contre-balancement des châssis s'effectue par un dispositif à contrepoids ou à ressorts, disposé à l'intérieur des montants du bâti.

Obs.: Fenêtre classique au Canada, la fenêtre à guillotine est souvent doublée d'une contre-fenêtre pour assurer une meilleure isolation contre le froid. On distingue la fenêtre à guillotine simple (single-hung window), dont un seul des deux châssis est mobile, de la fenêtre à guillotine double, dont les deux châssis sont mobiles (double-hung window).

Ouvertures

HS

fenêtre à la canadienne $f$ châssis à la canadienne $m$ Canadian sash, bottom-projected window

Fenêtre dont le châssis ouvre par projection horizontale vers l'intérieur, au moyen de biellettes.

Ouvertures

HS 
fenêtre à la française, fenêtre à battants $\mathrm{f}$ châssis à la française $m$ French window, casement window, French casement.

Fenêtre sur paumelles dont l'ouverture se fait par rotation sur un axe vertical vers l'intérieur de la pièce. Obs.: Fenêtre traditionnelle en France, elle peut comporter un ou deux vantaux (voir battant). On'dit de la fenêtre à la française qu'elle est ferrée (voir ferrer) sur le côté.

Ouvertures

HS

fenêtre à l'anglaise, fenêtre à battants $f$ châssis à 1 'anglaise $\mathrm{m}$ casement, outward-opening window

Fenêtre sur paumelles dont l'ouverture se fait par rotation sur un axe vertical vers l'extérieur de la pièce. Ouvertures

HS

fenêtre à l'australienne f châssis à l'australienne m Austral window

Fenêtre dont les deux châssis superposés ouvrent par projection horizontale, l'un vers l'intérieur et l'autre vers l'extérieur, et qui sont articulés par un système de biellettes.

Ouvertures

HS

fenêtre à l'italienne $f$ châssis à l'italienne $m$ top-hung window, italian sash, top projected window

Fenêtre dont le châssis ouvre par projection horizontale vers l'extérieur, au moyen de biellettes.

Obs. : En anglais on écrit aussi top hung window

Ouvertures

HS

fenêtre à soufflet $\mathrm{f}$ châssis-trémie $\mathrm{m}$ bottom-hung window, hopper light, hopper vent

Fenêtre sur paumelles dont la rotation se fait sur un axe horizontal situé le long de la traverse basse, et qui ouvre vers l'intérieur ou l'extérieur.

Obs.: Les détaillants de fenêtres donnent le nom de fenêtre à auvent (awning window) à la fenêtre à soufflet inversée, dont l'axe de rotation est situé le long de la traverse haute, et qui s'ouvre vers l'extérieur, comme un auvent.

Ouvertures

HS

fenêtre à tabatière, tabatière $\mathrm{f}$ skylight, rooflight Fenêtre pour toits en pente, dont le châssis ouvre vers l'extérieur, soit par projection, soit par rotation autour de l'axe horizontal supérieur.

Ouvertures

HS

fenêtre basculante $f$ châssis basculant $m$ horizontal pivoting window, horizontal pivot window, horizontal centre-hung window, tilting window

Fenêtre sur pivots, articulée en son centre pour permettre une rotation de $180^{\circ}$

Ouvertures

HS fenêtre coulissante $f$ châssis coulissant $m$ sliding window

Fenêtre dont le(s) châssis s'ouvre(nt) par translation horizontale sur un rail de roulement, ou qui sont suspendus à la traverse haute.

Ouvertures

HS

\section{fenêtre de doublage $\mathrm{f}$}

Voir contre-fenêtre.

\section{fenêtre de toit $\mathrm{f}$}

Voir fenêtre en toiture.

fenêtre en toiture, fenêtre de toit $f$ châssis de toiture m skylight, rooflight

«Elément de construction pour fermer une ouverture dans une toiture, destiné principalement à l'éclairage et consistant en un cadre et un vitrage.»

Obs. : La lucarne n'est pas une fenêtre de toit mais un ouvrage de maçonnerie faisant saillie. Le lanterneau et la fenêtre à tabatière sont les fenêtres de toit les plus courantes.

ISO 6707/1-1984

Ouvertures

HS

fenêtre oscillo-battante f tilt and turn window

Fenetre sur paumelles dont l'ouverture se fait par rotation sur un axe vertical et horizontal.

Ouvertures

HS

fenêtre panoramique, baie vitrée f picture window, window wall unit

«Fenêtre dont les grandes dimensions permettent l'effet du prolongement de la nature dans la maison.» LEMÉAC7 $1: 261$

Ouvertures

HS

fenêtre pivotante $f$ châssis pivotant $m$ pivoting sash, vertically pivoting window, vertical pivoting window, pivoted window

Fenêtre sur pivots, à rotation sur un axe vertical.

Obs.: Les versions récentes se ferment avec une manivelle.

Ouvertures

HS

fer $\mathrm{m}$ iron

«Métal résistant et malléable (.) surtout utilisé sous forme d'alliage, ou additionné de carbone pour constituer les aciers et les fontes.»

VIGAN92

Matériaux

CBR

fer à joints $m$ jointer, joint tool, finishing tool, striking tool

Outil de maçon, composé d'une tige ronde et muni d'une poignée, qui sert à former et à étendre le lit de mortier et à tirer et à finir les joints. 
Obs.: On distingue différents types de fers selon l'utilisation: à bordure pour cimentier, à rainures, concave, convexe, rond, en acier, en V, plat, rectangulaire; selon les matériaux : fer en acier, en bois ou en plastique.

Maçonnerie

MSP/MDR

fer à plat $m$

Voir langue-de-chat.

ferme f truss, roof truss

Structure principale, triangulaire, de la charpente de toit, conçue de façon à résister à toute déformation elle repose entièrement sur les murs extérieurs du bâtiment.

Toiture

MHL

ferme à un poinçon $\mathrm{f}$

Voir ferme simple.

ferme classique $f$ traditional truss (proposition)

Ferme composée de poutres de forte section et montée au moyen d'assemblage bois sur bois.

Obs.: Elle est formée de deux arbalétriers, d'un entrait et de différentes poutres qui assurent sa rigidité (aiguille pendante, contrefiche, poinçon, jambette, jambe de force, aisselier, etc.). Nous n'avons pas trouvé d'équivalent en anglais, cependant nous proposons traditional truss.

Toiture

MHL

ferme complexe f complex truss (proposition)

Ferme classique dont les arbalétriers doivent être soutenus afin de contrer l'effet de l'utilisation de pannes intermédiaires.

Obs.: En plus des arbalétriers et de l'entrait, elle comporte des poutres dont la fonction est de soutenir les nouds (contrefiche, jambette, faux-entrait, aisselier, aiguille pendante, jambe de force). Elle peut soutenir une ou plusieurs pannes et permet parfois de libérer suffisamment d'espace sous le comble pour pouvoir l'aménager. Nous n'avons pas trouvé d'équivalent en anglais, cependant nous proposons complex truss. Toiture

NHI

ferme moderne $f$ triangulated truss (proposition) Ferme dite triangulée, conçue selon les principes de la triangulation et calculée selon les règles de la résistance des matériaux : elle est composée de pièces de bois de faible section et montée au moyen d'assem blages métalliques.

Obs.: Elle est formée d'une membrure supérieure (jouant le même rôle que l'albalétrier d'une ferme classique) et d'une membrure inférieure (jouant le rôle de l'entrait) et d'un treillis (constitué de montants et/ou de diagonales, et parfois d'un poinçon). Nous n'avons pas trouvé d'équivalent en anglais, cependant nous proposons triangulated truss.

Toiture

MHL ferme simple, ferme à un poinçon $f$ king post truss Ferme classique ne portant pas de panne intermédiaire et dont les chevrons reposent uniquement sur la panne faîtière et sur les pannes sablières.

Obs. : Elle convient pour une portée maximum de 4 à 5 mètres. Si la portée est plus grande ou si la hauteur de la ferme nécessite l'emploi de pannes intermédiaires, on recourt à des fermes dont la structure est plus complexe (voir ferme complexe).

Toiture

MHL

ferrer $v t$ to fit locks and hinges

Articuler un ouvrant de fenêtre à son cadre dormant en posant des ferrures, pièces métalliques comme les paumelles et les pentures.

Ouvertures HS

feuillure f rebate, rabbet

Rainures pratiquées dans le châssis et destinées à recevoir le vitrage ou sur le linteau et les piédroits d'une baie afin de recevoir l'huisserie ou bâti dormant. Ouvertures

HS

fibre-ciment $m$ cement fibre

Terme générique pour les produits en amiante-ciment. Obs.: Composé de ciment et d'eau, le fibre-ciment offre une grande variété de produits, comme les plaques ondulées pour couverture, les tuyaux, les gaines carrées ou rectangulaires, les plaques planes ou les ardoises artificielles. Par déformation, les marques «Eternit» et «Everite» désignent souvent ces produits.

Toiture

JRS

fil à plomb, plomb $\mathrm{m}$ plumb line, plumb bob, plummet Outil composé d'une masse conique ou tronconique de métal, suspendue à un fil, et d'une plaquette carrée percée en son centre dont le côté est exactement égal au centre; il sert à vérifier et à contrôler la verticalité des ouvrages de maçonnerie.

Maçonnerie

MSP/MDR

finir les joints $v$ to repoint, to tuck-point

Remplir les joints ravalés à l'aide d'un fer à joints ou d'une truelle à jointoyer.

Maçonnerie

MSP/MDR

flexion $\mathrm{f}$ flexion

Contrainte s'exerçant perpendiculairement aux poutres et provoquant un fléchissement, ce qui entraîne un affaiblissement de la structure.

Toiture

MHL

gâcher $\mathrm{v}$ to mix, to temper

Incorporer de l'eau dans un liant, ou dans les constituants secs d'un mortier ou d'un béton, et mélanger jusqu'à obtention d'un mélange homogène. 
Obs.: On dit «gâcher du plâtre, du mortier, du béton». Matériaux

MDA

glaise $\mathrm{f}$

Voir argile.

gouge $m$ gouge

Outil de maçon composé d'un ciseau à tranchant courbe ou en $\mathrm{V}$ et d'un manche, qui sert à réaliser des moulures, des gorges, des cannelures.

Obs. : S'écrit parfois gouje.

Maçonnerie

MSP/MDR

\section{goutte d'eau $\mathrm{f}$}

Voir larmier.

granit, granite $\mathrm{m}$ granite

Roche éruptive dure, formée de cristaux de feldspath, de quartz et de mica, qui a la particularité de prendre un beau poli.

Obs.: Le granit est employé en construction, car il a la réputation d'être inaltérable. Pouvant prendre des teintes diverses (blanc, gris clair, rose, gris-vert), il est essentiellement utilisé comme matériau ornemental.

Matériaux

MDA

granite $\mathbf{m}$

Voir granit.

granulat $m$ aggregate

Matériau granulaire inerte - sable, gravier, galets, cailloux, etc. - que l'on mélange à un liant pour faire un mortier ou un béton.

Obs.: On distingue les granulats naturels (calcaires, siliceux, silico-calcaires) et les granulats artificiels (argile, schiste expansés, etc.).

On emploie abusivement agrégat comme synonyme de granulat.

Matériaux

CBR

gratte f mortar ho

Outil muni d'une lame large avec deux orifices, qui sert à malaxer manuellement des petites quantités de ciment ou de mortier.

Maçonnerie

MSP/MDR

\section{gratton $m$ devil float}

Outil composé d'une semelle en bois munie d'une poignée et de pointes d'acier dures, qui sert à gratter la surface des enduits afin d'en assurer la finition.

Maçonnerie

MSP/MDR

grès $m$ sandstone

«Roche silicieuse, résultant de la sédimentation du sable, utilisée dans la maçonnerie (.)»

BROCHEN95

Matériaux

MDA grès cérame $m$ stoneware

Céramique dure, imperméable et opaque, obtenue par la cuisson à température élevée d'un mélange de silice et d'argile.

Obs, : De nombreux carreaux, en particulier ceux qui sont utilisés pour les revêtements extérieurs, sont fabriqués en grès cérame.

Matériaux

MDA

guillotine, fendeuse f masonry cutting machine, splitter Machine dotée de deux lames, l'une supérieure et l'autre inférieure, qui sert à couper un élément de maçonnerie.

Obs.: Cette machine peut être hydraulique ou pneumatique.

Maçonnerie

MSP/MDR

gypse m gypsum

«Minéral appelé communément "pierre à plâtre" constitué essentiellement de sulfate de calcium déshydraté $\mathrm{CaSO}_{4} 2 \mathrm{H}_{2} \mathrm{O}$. Il est introduit au cours de la mouture de la plupart des ciments pour en régulariser la prise.

Osb.: On retrouve l'adjectif gypseux et le substantif gypserie (panneau ornemental à base de plâtre).

CILF76:98

Matériaux

CBR

hachette $f$ hatchet

Petite hache dotée d'un taillant et d'une tête ayant les fonctions d'un marteau.

Obs.: Les formes changent selon le corps des métiers: hachette de briqueteur, hachette de plâtrier, hachette de maçon.

Maçonnerie

MSP/MDR

\section{huisserie $f$}

Voir bâti dormant.

isolation $f$ insulation

«Réduction ou suppression du cheminement d'un phénomène (électrique, acoustique, calorifique) entre deux milieux, par l'interposition d'un matériau dont c'est la fonction principale: isolant phonique, isolant électrique, isolant thermique.»

VIGAN92

Ouvertures

HS

isolation acoustique $\mathrm{f}$

Voir isolation phonique

isolation phonique, isolation acoustique $f$ sound proofing, sound insulation

Installation ayant pour but d'éviter la propagation des sons de l'extérieur d'un bâtiment vers l'intérieur, ou l'inverse.

Ouvertures

HS 
isolation thermique $f$ thermal insulation, heat insulation

«Ensemble des techniques mises en œuvre pour limiter les déperditions de chaleur de l'intérieur vers l'extérieur.»

Obs.: Une bonne isolation thermique est efficace aussi dans le sens inverse, où elle améliore le confort d'été, en contribuant au maintien d'une température intérieure inférieure à la température extérieure.

VIGAN92

Ouvertures

HS

jambage, piédroit $\mathrm{m}$ window post, jamb

Élément de maçonnerie vertical situé de part et d'autre d'une baie pour en soutenir le linteau. Ouvertures

HS

jambe de force $f$ strut (of a truss), brace (of a truss), stay

Poutre oblique, située entre le noud inférieur de l'arbalétrier et le mur ou une poutre du plancher de l'étage inférieur qui remplace parfois l'ensemble jambette-aisselier.

Obs. : Elle passe au travers de l'entrait qui, dans ces conditions, est composé de pièces parallèles et forme donc un entrait moisé.

Toiture

MHL

jambette $f$ side post, princess post

Courte poutre verticale qui supporte les nœuds les plus bas d'une ferme complexe soutenant plusieurs pannes intermédiaires.

Obs.: Elle s'appuie sur l'entrait et travaille en compression (contrairement à l'aiguille pendante, qui travaille en traction).

Toiture

MHL

jauge, jauge à mesurer, jauge de briqueteur $\mathrm{f}$ piedde-roi $m$ gauge rod, story pole, story rod

Bâton en bois sur lequel sont indiquées la hauteur des ouvertures et des rangées de briques, et qui sert de repère pendant la construction $d^{\prime}$ 'un mur.

Maçonnerie

MSPMDR

jauge à mesurer $\mathrm{f}$

Voir jauge.

\section{jauge de briqueteur $\mathrm{f}$}

Voir jauge.

jet d'eau, rejet d'eau $m$ weatherboard, sill flashing Extrémité basse d'un châssis dont la forme arrondie et en saillie permet d'éviter la pénétration d'eau entre le dormant et la traverse d'appui du châssis.

Ouvertures

HS jointer, jointoyer $v$ to point, to tool, to strike, to finish Remplir les joints et comprimer le mortier entre les éléments de maçonnerie pour les sceller et éviter la pénétration d'humidité.

Maçonnerie

MSP/MDR

\section{jointoyer $\mathrm{v}$}

Voir jointer

lame d'air $\mathrm{f}$ matelas d'air $\mathrm{m}$ airspace

Espace d'air déshydraté ou de gaz inerte enfermé de manière étanche entre deux feuilles de verre pour en améliorer l'isolation.

Obs.: L'isolation thermique varie avec l'épaisseur de la lame d'air emprisonnée.

Ouyertures

HS

langue-de-chat $\mathrm{f}$ fer à plat $\mathrm{m}$ calking trowel Petite truelle à lame fine et à bout arrondi, qui sert à remplir un joint.

Obs. : Elle existe en plusieurs dimensions.

Maçonnerie

MSP/MDR

lanterneau, châssis de toiture $m$ skylight, lantern light Dispositif constituant une prise de jour en toiture et ayant la forme de petits dômes translucides en plexiglas, que l'on peut soulever pour l'aération.

Obs. : Ils peuvent être fixes ou ouvrants.

Ouvertures

HS

larmier $\mathrm{m}$ goutte d'eau $\mathrm{f}$ weather moulding, drop apron, drip cap

Moulure creuse destinée à arrêter les filets d'ean et à les faire tomber directement sur le sol, en gouttes ou larmes.

Obs.: Il peut être situé sous l'appui d'une baie pour empêcher le ruissellement des eaux le long du mur; sous la traverse basse des ouvrants pour empêcher l'eau d'infiltration; au sommet de la fenêtre pour empêcher l'eau de ruisseler sur les carreaux.

Ouvertures

HS

lattis m latice

Ensemble des lattes parallèles, espacées ou jointives, qui sont clouées sur un pan de bois ou sur un chevronnage de toit.

Toiture

JRS

laye $f$ axhammer, face hammer

Outil de frappe muni de deux têtes dont l'une est tranchante et non dentée, et l'autre carrée, qui sert à couper la pierre.

Obs. : S'écrit aussi laie

Maçonnerie

MSP/MDR 
liane $\mathrm{f}$

Voir lisseuse

\section{liant $m$ binder}

«Matière qui a pour propriété (.) de passer de l'état liquide ou plastique à l'état solide, pour lier, c'està-dire assembler par collage des matériaux inertes: sables, cailloux, granulats divers, fibres, bois, métaux, matières plastiques, etc.»

Obs.: On distingue deux grands types de liants: les liants minéraux (liant hydraulique, liant gypseux, liant aérien) et les liants organiques (liant hydrocarboné, liant noir, liant à base de résine)

VIGAN92

Matériaux

CBR

ligne à briqueteur $\mathrm{f}$ cordeau, cordeau d'alignement m mason's line

Long fil tendu par le maçon pendant la construction d'un mur pour assurer l'alignement des rangées de briques.

Maçonnerie

MSP/MDR

linteau $m$ traverse dormante $f$ lintel

Pièce horizontale de bois, de pierre ou de métal, supportée par les jambages et formant la partie supérieure de la baie.

Ouvertures

HS

lisseuse, taloche souple, liane $f$ plâtroir $m$ plastering trowel, steel trowel

Outil en bois, en métal ou en plastique consistant en un plateau, une poignée et une lame semi-rigide qui sert à étaler et égaliser de l'enduit, du plâtre, du mortier ou des crepis.

Maçonnerie

MSP/MDR

liteau m splat

Latte carrée ou rectangulaire qui, clouée horizontalement sur les chevrons avec un intervalle égal au pureau des tuiles, sert à accrocher les tuiles et les ardoises.

Toiture

JRS

malaxer $\mathrm{v}$ to mix

Brasser les ingrédients d'un mortier ou d'un béton avec de l'eau, à la main ou à l'aide d'un malaxeur à mortier ou d'une bétonnière.

Maçonnerie

MSP/MDR

malaxeur à mortier $\mathrm{m}$ mortar mixer, mortar mill

Cuve dotée de palettes animées, qui sert à mélanger les composants du mortier.

Maçonnerie

MSP/MDR marbre m marble

Roche calcaire très dure, souvent veinée de couleurs varieses, qui se compose essentiellement de carbonate de calcium cristallisé (calcite) ou de carbonate double de calcium et de magnésium (dolomie) et qui a la particularité de prendre un beau poli.

Obs.: Dans le bâtiment, on utilise le marbre surtout comme revêtement des sols et placage muraux, intérieurs ou extérieurs, mais aussi pour les marches, les plinthes, les tablettes et divers autres éléments de décoration.

Les différents types de marbres sont classés en fonction de leur couleur dominante: blancs (fond crème ou bleuté), beiges, bleus, noirs (fond noir, ramagé ou veiné), gris, jaunes, roses, rouges, verts et violets.

Matériaux

MDA

marne f marl

Mélange naturel d'argile et de calcaire, dont certaines compositions sont directement utilisables pour la fabrication des ciments naturels.

Matériaux

CBR

\section{marteau m hammer}

Outil de frappe composé d'un manche et d'une tête métallique, généralement en acier dur trempé qui s'utilise sur un outil de coupe ou de formage (ciseau, burin), soit sur un accessoire à enfoncer (clou), soit en frappe directe.

Obs.: La forme de l'outil varie selon l'utilisation.

Maçonnerie

MSP/MDR

marteau à brique $\mathrm{m}$

Voir marteau de maçon

marteau de maçon, marteau à brique m bricklayer's hammer, brick hammer

Outil de frappe dont une panne est carrée et l'autre se termine en pointe, qui sert à couper ou à tailler les briques.

Maçonnerie

MSP/MDR

marteau pneumatique $\mathrm{m}$ jack hammer, pneumatic hammer

Outil de frappe muni d'un compresseur, qui sert à pratiquer des ouvertures dans le béton.

Maçonnerie

MSP/MDR

martelet $\mathbf{m}$ tile hammer

Outil de frappe à tête carrée et à panne effilée en pic, qui sert à couper les unités de maçonnerie légères.

Maçonnerie

MSP/MDR

masse de maçon $\mathrm{m}$ sledge hammer

Gros marteau à tête carrée lourde et à manche court, qui sert à enfoncer les pieux, à frapper sur les burons ou à dégrossir des matériaux bruts.

Maçonnerie

MSP/MDR 


\section{matelas d'air $\mathrm{m}$}

Voir lame d"air

matériaux m materials

«(T)oute matière et tout elément utilisé dans la construction d'un bâtiment ou d'un ouvrage.»

Obs. : Le mot est souvent employé avec un qualificatif décrivant une caractéristique primordiale ou une fonction: les matériaux de revêtement, les matériaux de couverture, etc. Selon les auteurs, matériaux de construction désigne tous les éléments destinés à être assemblés pour édifier un ouvrage : briques, parpaings, pierres, etc. Ce terme est plus spécifique dans le sens où il n'englobe pas un matériau comme le plastique ou l'acier (qui entrent sous l'appellation plus générique de materiaux).

BROCHEN95

Matériaux

CBR

membrure inférieure f bottom chord, bottom member Pièce de bois jouant le rôle d'entrait dans une ferme moderne.

Toiture

MHL

membrure supérieure f top chord, top member Pièce de bois jouant le rôle d'arbalétrier dans une ferme moderne.

Toiture

MHL

meneau $\mathrm{m}$ mullion, transom (horizontal), muntin (vertical)

Chacune des pièces horizontales et verticales qui divisent le vitrage d'une croisée.

Obs.: Le meneau peut être en bois (petit-bois) ou en métal. Le terme est aussi employé pour désigner le montant dormant séparant plusieurs châssis d'un ouvrage composé.

Ouvertures

HS

métal m metal

«Désigne une famille de matériaux remarquables par leurs propriétés de résistance mécanique et leur conductivité de la chaleur et de l'électricité (acier, aluminium, argent, bronze, cuivre, étain, fer, fonte, laiton, nickel, platine, plomb, or, zinc, etc.).»

Obs.: On adapte les métaux aux caractéristiques recherchées en constituant des alliages.

BROCHEN95

Matériaux

CBR

minéral m mineral

«Matière non organique, solide et inerte à l'état naturel.» Obs.: Les minéraux sont soit amorphes (sans constituants organisés) soit cristallisés.

VIGAN92

Matériaux

CBR moellon $\mathrm{m}$ rubble

Petit bloc de pierre calcaire, brut ou taillé, utilisé dans la construction des murs en pierre.

Obs.: On distingue différents types de mœllons en fonction de leurs dimensions, leur taille et leur position dans la maçonnerie. Le mœllon brut ou moellon bloqué, de petite dimension et non équarri, sert comme blocage à l'intérieur de la maçonnerie; le mœllon de plat est posé sur son lit, c'est-à-dire sa face la plus grande; et le mœllon ébauché est taillé uniquement sur sa face de parement.

Matériaux

MDA

montant (1) m upright, stile

Chacune des pièces verticales qui composent le cadre dans l'ouvrant ou le dormant d'une fenêtre.

Ouvertures

HS

montant (2) m upright, vertical member

Élément vertical du treillis d'une ferme triangulée.

Toiture

MHL

montant battant $\mathrm{m}$

Voir montant de battement.

montant charnier, montant de rive, montant ferré $m$ hanging stile

Montant de fenêtre qui porte les ferrures et les organes de rotation tels que les charnières et les paumelles.

Ouvertures

HS

montant de battement, montant battant $\mathrm{m}$ closing stile, slamming stile

«Montant d'un vantail qui, à la fermeture, vient soit s'appuyer sur le bâti dormant, soit joindre le montant correspondant du vantail voisin.»

QUILLET59:638

Ouvertures

HS

\section{montant de rive $\mathrm{m}$}

Voir montant charnier.

\section{montant ferré $m$}

Voir montant charnier.

\section{mortier m mortar}

Mélange de gravier, de sable, d'eau et d'un liant, servant à solidariser les différents éléments de maçonnerie ou à réaliser des enduits, des joints, des chapes, des scellements.

Obs.: Selon la dimension des grains de sable qu'i contient, un mortier est dit fin, moyen ou gros. On dit «étendre le mortier», «épandre le mortier», «régaler le mortier» et «buriner le mortier».

Matériaux

MDA 
mortier bâtard $\mathrm{m}$ cement-lime mortar, lime cement mortar

Mortier utilisé pour la maçonnerie ou dans les enduits dont le liant est composé en proportions variables, généralement en quantités égales, de chaux et de ciment.

Obs.: Il existe de nombreux synonymes en anglais. Le choix des équivalents s'est fait en fonction de leur fréquence d'utilisation. S'écrit aussi en anglais cement/ lime mortar.

Matériaux

MDA

mortier de bourrage $m$ dry pack

«Mortier très sec, mis en place par bourrage, servant à remplir les espaces peu accessibles.»

PARÉ93

Matériaux

MDA

mortier de chaux $m$ lime mortar

Mortier dont le liant est de la chaux, éteinte ou hydraulique.

Matériaux

MDA

mortier de ciment $m$ cement mortar

Mortier dont le liant est du ciment.

Matériaux

MDA

mortier gras $\mathrm{m}$ fat mortar

Mortier contenant une forte proportion de liant, chaux ou ciment.

Matériaux

MDA

mortier maigre m lean mortar

Mortier contenant une faible proportion de liant, chaux ou ciment.

Matériaux

MDA

niveau à bulle $\mathrm{m}$

Voir niveau à bulle d'air.

niveau à bulle d'air, niveau à bulle $m$ spirit level Longue règle renfermant un ou deux tubes en verre contenant un liquide et une bulle gazeuse, qui sert à indiquer l'horizontalité ou la verticalité d'un ouvrage. Maçonnerie MSP/MDR

niveau de maçon $m$ plumb rule

Niveau en forme d'équerre en $\mathrm{A}$, au sommet duquel est accroché un fil à plomb, qui sert à aligner les blocs de béton pour établir l'horizontalité d'une surface. Maçonnerie MSP/MDR

noue $f$ valley

Intersection de deux pans rentrants de couverture.
Obs.: Par extension, on appelle également noue, la bande de zinc ou de plomb fixée à cette intersection. La contrepartie est l'arêtier.

Toiture

JRS

\section{ouvrant m}

Voir châssis.

panne f purlin

Poutre horizontale d'une charpente de toiture, parallèle à l'égout, en bois ou en métal, qui repose sur les arbalétriers et qui reporte sur les fermes les charges transmises par la couverture et les chevrons.

Toiture

MHL

\section{panne à mortier $\mathbf{f}$}

Voir boîte à mélange.

panneau, panneau de sous-toiture $\mathrm{m}$ panel

Plan de matériaux rigide supportant la couverture et souvent fait de particules de bois latté, de contreplaqué ou de feuilles métalliques ondulées.

Obs.: Les feuilles métalliques sont en général associées à des isolants thermiques en mousse rigide.

Couverture

JRS

panneau de sous-toiture $m$

Voir panneau.

panne faitière fridge beam, ridge piece, ridge purlin Panne située sous le faîte du toit, et qui supporte l'extrémité supérieure des chevrons.

Toiture

MHL

panne intermédiaire $f$ intermediate purlin Panne située entre la panne sablière et la panne faîtière. Toiture

MHL

panne sablière $f$ eaves purlin

Panne située à la base de la toiture, au-dessus du mur gouttereau supportant l'égout; assemblée aux arbalétriers, elle porte les pieds des chevrons.

Toiture

MHL

parclose $\mathrm{f}$ glass stop, glazing bead, glazing fillet

Baguette de bois ou de métal servant au maintien des vitrages dans les feuillures de châssis.

Ouvertures

HS

paumelle $f$ hinge

«Ferrure de rotation, reliant un battant au cadre dormant, ou à la maçonnerie et lui permettant de pivoter. La paumelle se compose de deux éléments, l'un mâle, l'autre femelle.»

BROCHEN95

Ouvertures

HS 


\section{pente f pitch}

Inclinaison d'un versant de toiture, qui varie selon les matériaux de couverture, le climat et les styles architecturaux de la région.

Obs. : On confond souvent versant, pan et pente. Dans le domaine de la toiture, pan et pente sont pratiquement synonymes: un pan est une surface plane, une pente est une surface plane inclinée. Toutefois, un versant peut comporter plusieurs pans (et donc, plusieurs pentes). Dans le cas de la toiture à pignons, les trois termes sont synonymes.

Toiture

JRS /MHL

pic $m$ pick

Marteau doté d'une ou de deux pannes pointues, qui sert à faire les parements éclatés.

Maçonnerie

MSP/MDR

pièce d'appui, traverse d'appui f bottom member

Traverse inférieure du bâti dormant d'une fenêtre.

Obs.: On l'emploie aussi pour désigner la traverse inférieure du châssis mobile.

Ouvertures

HS

\section{pied-de-roi $m$}

Voir jauge.

piédroit $m$

Voir jambage.

pierre $\mathrm{m}$ stone

«(E)nsemble des blocs de matière minérale, taillés dans des bancs de roche ou ramassés à l'état naturel, utilisés comme matériaux de base de très nombreux types de maçonnerie.»

Obs.: Une pierre est caractérisée par sa dureté relative, sa difficulté de taille, sa structure, sa masse volumique, sa résistance à l'écrasement, la texture de sa cassure et sa couleur. On distingue différents types de pierres, par exemple, la pierre artificielle (béton), la pierre à bâtir (granite, marbre, etc.), la pierre à chaux, la pierre à huile, la pierre marbrière, la pierre pelliculaire, la pierre à plâtre (gypse), la pierre-sèche, etc. BROCHEN95

Matériaux

CBR

pierre à bâtir $\mathrm{f}$ building stone

Nom générique désignant l'ensemble des pierres, brutes ou taillées, utilisées dans le bâtiment.

Matériaux

MDA

pierre de taille $f$ ashlar, cut stone

Bloc de pierre naturelle taillé à l'outil suivant des formes et des dimensions préalablement déterminées en fonction de la place qu'il doit occuper dans l'ouvrage.

Matériaux

MDA pierre marbrière $\mathrm{f}$ marble-like stone

Pierre calcaire dont l'apparence et la dureté sont semblables à celles du marbre.

Matériaux

MDA

pige f measuring rod

Baguette d'une dimension déterminée qui sert d'unité de longueur répétitive dans des tracés et de contrôle des profondeurs et d'épaisseur des enduits frais.

Maçonnerie

MSP/MDR

pignon $m$ gable

Mur extérieur qui porte les pannes et dont les contours épousent la forme des versants de la toiture par opposition aux murs gouttereaux, situés sous les égouts des pans de toiture.

Obs.: À l'origine, le pignon ne désignait que la partie de mur triangulaire délimitée par les toitures; cette partie est aujourd'hui nommée pointe de pignon.

Toiture

MHL

pince à brique $\mathrm{f}$ brick clamps

Outil constitué de deux branches qui se resserrent pour saisir et serrer les briques afin de les transporter. Maçonnerie MSP/MDR

pistolet à calfeutrer $m$ caulking gun

Outil constitué d'un réservoir, d'une buse et d'une poignée-crosse, qui sert à projeter un produit par pression manuelle ou automatique.

Maçonnerie

$\mathrm{MSP} / \mathrm{MDR}$

pivot $\mathrm{m}$ pivot hinge

Organe de rotation des fenêtres basculante et pivotante, constitué de deux éléments: une pièce cylindrique permettant à une autre de tourner sur son axe.

Ouvertures

HS

plâtre m gypsum plaster

«Sulfate de calcium calciné et réduit en poudre employé délayé avec de l'eau, pour cimenter les pierres, faire des enduits, mouler des statues, etc. Il est le résultat du traitement du gypse.»

Obs.: On dit "gâcher le plâtre». Les plâtres sont tous les ornements faits en plâtres. On connaît l'usage du plâtre depuis la Haute Antiquité (2 800 ans av. J.-C.).

QUILLET68: 1716

Matériaux

CBR

plâtroir m

Voir lisseuse.

plomb m

Voir fil à plomb. 
poinçon $\mathrm{m}$ king post

Poutre verticale centrale d'une ferme classique, dont le rôle est de faciliter l'assemblage des arbalétriers à la tête de la ferme et de soutenir l'entrait, qui a tendance à fléchir sous son propre poids.

Obs. : Il n'intervient pas dans la stabilité de l'ensemble de la ferme, puisque les arbalétriers et l'entrait forment un triangle, c'est-à-dire un système indéformable (à condition que les assemblages soient solides).

Toiture

MHL

pointe $f$

Voir ciseau de maçon.

pointe de maçon $f$ point chisel

Outil en acier de carbure doté d'une extrémité en pointe, qui sert à enlever des petits morceaux de pierre. Maçonnerie

MSP/MDR

portée $f$ span

Distance séparant deux points d'appui consécutifs d'une poutre, d'une solive ou de tout élément porteur. Toiture

MHL

poutre $f$ beam

Toute importante pièce de bois, de métal ou de béton armé, selon la structure de la charpente, servant de support à une autre pièce de charpente ou à la toiture. Obs. : En français, chaque poutre porte un nom particulier selon son emplacement et sa fonction, nom qu'elle tire aussi, souvent, de la tradition. En anglais cependant, seule la fonction détermine le nom de la poutre (voir observations à traction et à compression). Toiture MHL

pureau $m$ gauge

Partie visible d'un matériau de couverture, comme l'ardoise ou la tuile, qui n'est pas recouverte par les éléments du rang supérieur.

Toiture

JRS

rabot à mortier $\mathrm{m}$

voir rabot de maçon.

rabot de macon, rabot à mortier m rake, larry

Sorte de pelle qui sert à humidifier la chaux et à gâcher les bétons et les mortiers.

Maçonnerie

$\mathrm{MSP} / \mathrm{MDR}$

rabotin $\mathrm{m}$ drag, comb

Outil à huit lames finement dentées et montées sur une semelle de bois à poignée, qui sert à ragréer les façades de pierre.

Maçonnerie

MSP/MDR raclette $f$ scraper

Outil doté d'un rebord de caoutchouc semi-rigide et d'un manche, qui sert à faire pénétrer dans les joints les coulis de mortier de jointoiement.

Maçonnerie

MSP/MDR

ragréer $\mathrm{v}$ to smooth

Supprimer les irrégularités d'une surface afin de la rendre plane avant d'appliquer un enduit ou un revêtement.

Maçonnerie

MSP/MDR

ravaler $\mathrm{v}$ to rake

Enlever ou gratter le mortier des joints ou des murs lors des travaux de finition.

Maçonnerie

MSP/MDR

règle de maçon $\mathrm{f}$ straightedge

Longue règle en bois ou en métal, qui sert à tirer des chapes, à prendre des nivaux, à vérifier la planéité ou à égaliser les enduits avant le talochage.

Maçonnerie

MSP/MDR

rejet d'eau $m$

Voir jet d'eau.

repère $m$ screed

Baguette qui sert de guide d'épaisseur pour l'application d'un enduit.

Maçonnerie

MSP/MDR

rive $f$ edge

Ligne délimitant divers plans, comme une lame de parquet ou une tôle, avec le vide.

Obs.: En toiture, la rive délimite les côtés inclinés d'un pan de couverture. Elle est différente du faîte et des égouts. Elle peut être droite, donc parallèle à la ligne de plus grande pente, ou au contraire, biaise. Lorsque la toiture n'a qu' un versant et qu'elle est donc sans faîtage, son bord supérieur est appelé rive de tête. Couverture JRS

roche $\mathrm{f}$ rock

«Minéral de composition et de structure homogène, qui se présente en masses compactes. Plus généralement, désigne toute matière dure extraite du sol pour être utilisée comme matériau de construction.» Obs. : En géologie, on classe les roches en fonction de leur origine ou de leur formation, en roches endogènes, sédimentaires et métamorphiques. La roche constitue le générique qui englobe les pierres, le granite, les sables, les galets, les argiles, les calcaires, les bitumes et asphaltes, etc. qui sont, eux, des spécifiques. VIGAN92

Matériaux

CBR 
ruban à mesurer de maçon m mason's rule, steel tape

Instrument de mesure fait en bois ou en fer.

Obs.: Lorsqu'il est en bois, il se replie; lorsqu'il est en fer, il se roule sur lui-même.

Maçonnerie

MSP/MDR

sablière $\mathrm{f}$

Voir panne sablière

scie mécanique à maçonnerie, scie motorisée à maçonnerie f masonry saw

Scie munie d'une lame abrasive, dotée d'un disque tournant, qui sert à couper la pierre ou les blocs, ou à pratiquer une ouverture sur un mur.

Maçonnerie

MSP/MDR

scie motorisée à maçonnerie $\mathbf{f}$

Voir scie mécanique à maçonnerie.

scie sauteuse f jigsaw, saber saw

Scie munie d'une lame qui fait un mouvement vertical, perpendiculaire à la matière, et qui sert à faire des supports d'arche.

Maçonnerie

MSP/MDR

set à brique $m$

Voir ciseau à brique.

spatule $f$ filling knife, broad knife

Outil constitué d'un manche et d'un fer plat souple, en forme de petite pelle, qui sert à appliquer du mastic ou à enlever de la peinture.

Maçonnerie

MSP/MDR

support à ligne $m$ tingle

Cale maintenant un cordeau d'alignement lorsqu'un mur en cours de montage est très long, afin d'éviter les écarts d'alignement provoqués par l'action du vent sur le cordeau.

Maçonnerie

MSP /MDR

support de ligne $f$ line twig

Latte ou réglette horizontale avec des rebords sur lesquels on fixe les cordeaux d'alignement.

Maçonnerie

MSP/MDR

survitrage m secondary glazing, overglazing

Vitrage appliqué à l'intérieur contre le vitrage d'une baie pour en améliorer les caractéristiques thermiques et phoniques.

Obs.: Le survitrage peut être réalisé au moyen de vitres, ou de films ou de panneaux de matière plastique; dans ce dernier cas, on parle de survitrage plastique pour le distinguer du survitrage vitré. Le survitrage est différent du double vitrage car dans le survitrage, la lame d'air emprisonnée entre les deux vitres est immobile mais non déshydratée, contrairement au double vitrage.

Ouvertures

HS

tabatière $\mathrm{f}$

Voir fenêtre à tabatière.

table à mortier f mortarboard, mortar spot board, spot board, fat board

Planche en bois ou de contre-plaqué, montée sur deux pièces de bois, qui sert à déposer le mortier lors du travail.

Maçonnerie

MSP /MDR

\section{tablette d'appui $f$}

Voir appui

taloche f float, darby

Planchette de bois munie d'un manche, qui sert à porter et à appliquer les enduits et le plâtre sur les plafonds et sur les murs.

Maçonnerie

MSP/MDR

talocher $\mathrm{v}$ to float

Étaler et repartir un enduit à l'aide d'une taloche.

Maçonnerie

MSP/MDR

taloche souple

voir lisseuse.

tamis m sieve

Bac à fond grillagé qui sert à séparer les gros éléments des petits dans un mélange sec.

Maçonnerie

$\mathrm{MSP} / \mathrm{MDR}$

\section{tavaillon $\mathrm{m}$}

Voir bardeau.

\section{tendeur à ligne $\mathrm{m}$}

Voir coin.

théodolite $\mathrm{m}$ theodolite

Instrument ressemblant à un niveau à lunette, qui sert à déterminer le niveau et la hauteur.

Maçonnerie

MSP/MDR

toiture f roofing

Ensemble de tous les éléments composant le recouvrement et la couverture d'un bâtiment, aussi bien les matériaux de couverture comme les ardoises, les tuiles ou le zinc, que leurs supports.

Obs.: Par déformation de sens, la toiture peut aussi inclure d'autres parties, tels que la structure composée 
par la charpente et le voligeage, le volume des combles et même les lucarnes ou les verrières. La charpente, et donc aussi le support de couverture de toit, varient entre autres, selon son architecture. Toiture

JRS

toiture inclinée f pitched roof

«Toiture dont l'inclinaison est généralement supérieure à 10 (environ $15 \%$ ), 》 ISO6707/1: 13

Toiture

MHL

tôle $\mathrm{f}$ iron roof

Matériau de couverture, généralement en acier, dont on obtient les feuilles par laminage.

Obs.: Parmi les types de tôle les plus courants en couverture, on trouve: la tôle d'acier inoxydable; la tôle nervurée; la tôle d'aluminium; la tôle électrozinguée, dont l'acier est recouvert de zinc par électrolyse; la tôle émaillée recouverte d'émail cuit au four; la tôle galvanisée trempée dans du zinc en fusion; la tôle laquée, plane, recouverte de couches de protection contre la corrosion et présentée sous des couleurs variées; et la tôle ondulée qui est légère et économique.

Toiture

JRS

traction $\mathrm{f}$ traction

Contrainte s'exerçant dans le sens longitudinal d'une poutre; elle provoque un allongement ou une extension. Obs.: En anglais, les poutres travaillant en traction sont nommés tie ou encore brace (tirant).

Toiture

MHL

traverse f strut, crosspiece

«Pièce qui constitue un des côtés horizontaux des ouvrages et parties d'ouvrages.»

Obs.: Une traverse désigne tout élément horizontal, qu'il appartienne au bâti dormant ou au châssis de la fenêtre.

QUILLET59

Ouvertures

HS

traverse basse $\mathrm{f}$ bottom rail

Traverse inférieure d'un châssis.

Ouvertures

HS

traverse d'appui $\mathrm{f}$

Voir pièce d'appui.

traverse dormante $f$

Voir linteau.

traverse haute f top rail

Traverse supérieure d'un châssis.

Ouvertures

HS triangulation $\mathrm{f}$ triangulation

«Utilisation du caractère indéformable du triangle comme principe de base de conception des éléments de structure des charpentes, des fermes, etc.»

VIGAN92

Toiture

MHL

truelle f trowel

Outil doté d'une lame et d'un manche, qui sert à poser ou à dresser un enduit.

Obs.: Au Canada, deux modèles sont utilisés, à savoir la truelle London (à épaule arrondie) et la truelle Philadelphia (à épaule carrée). Le choix de l'une ou de l'autre est personnel, les deux modèles servant à exécuter la même tâche.

Maçonnerie

MSP/MDR

truelle à bout carré

Voir truelle de plâtrier

truelle à brique $f$ brick trowel

Truelle de forme ovoïde qui sert à monter au plâtre les cloisons en briques.

Maçonnerie

$\mathrm{MSP} / \mathrm{MDR}$

truelle à jointoyer $f$ pointing trowel

Truelle à lame pointue ou carrée, qui sert à travailler les mortiers.

Maçonnerie

$\mathrm{MSP} / \mathrm{MDR}$

truelle briqueteuse, briqueteuse $\mathrm{f}$ buttering trowel

Truelle plus petite que la truelle à brique, qui sert à appliquer le mortier sur la brique ou graisser la brique, avant de la poser.

Obs. : En anglais, «graisser la brique» se dit to butter a brick.

Maçonnerie

MSP/MDR

truelle carrée $\mathrm{f}$

Voir truelle de plâtrier.

truelle d'angle $f$ angle trowel

Truelle à lame courbée à un angle d'environ 90 degrés, qui sert à finir les coins.

Maçonnerie

MSP/MDR

truelle de plâtrier, truelle carrée, truelle à bout carré $f$ square trowel

Truelle à lame flexible et à bout rond, carré ou rectangulaire, qui sert à travailler le plâtre.

Maçonnerie

MSP/MDR

trumeau m pier

Partie d'un mur d'une cloison comprise entre deux baies rapprochées.

Ouvertures

HS 
tuile f tile

Matériau de recouvrement, en forme de plaquette rigide obtenue par le moulage ou le pressage d'une matière manufacturée, puis cuite.

Obs.: La tuile peut être plane, cintrée, nervurée ou ondulée. Généralement en terre cuite, elle peut aussi être en béton, en verre ou en métal. On la choisit en fonction de la pente du toit et du style de la région concernée.

Toiture

JRS

valeur de recouvrement $m$ roofing value

Limite de sécurité, issue de calculs effectués dans le but d'empêcher l'eau de pluie de remonter jusqu'au bord supérieur d'un toir en pente.

Obs.: Ces calculs se font en fonction de la pente de la couverture, de la zone climatique de la région concernée, de la longueur du rampant et du mode de fixation de la couverture.

Toiture

JRS

vantail $m$

Voir battant.

verre isolant $m$

Voir double vitrage.

vide joints $m$

Voir bédane.

vitrage $m$ glazing, window glazing

Désigne à la fois l'ensemble des vitres d'une baie et l'action de mettre en place les vitres.

Obs. : Le choix d'un vitrage est fonction des caractéristiques attendues, concernant non seulement sa transparence à la lumière, mais aussi son pouvoir d'isolation thermique et phonique, ses caractéristiques relatives à la sécurité et à la protection solaire. Un vitrage est surtout caractérisé par son épaisseur.

Ouvertures

HS

vitrage isolant $m$

Voir double vitrage.

Liste français-anglais

$\begin{array}{lrl}\text { acier } & \begin{array}{r}\text { steel } \\ \text { additif }\end{array} & \text { appui } \\ \text { adjuvant } & \text { voir adjuvant } & \text { arbalétrier } \\ \text { aiguille } & \text { admixture, additive } & \\ \text { aiguille pendante } & \text { tie, brace } & \text { ardoise } \\ \text { aisselier } & \text { voir aiguille } & \text { arêtier } \\ \text { allège } & \text { brace } & \text { argile } \\ \text { alliage } & \text { apron wall, breast, window breast, breast wall } & \text { asphalte } \\ \text { aluminium } & \text { alloy } & \text { baie } \\ \text { aplainissoir } & \text { aluminum } & \text { baie vitré } \\ & \text { float } & \text { bardeau }\end{array}$

vitre $f$ carreau $m$ pane, pane of glass, window pane «Plaque de verre dont sont munies les fenêtres.» Obs.: Carreau évoque davantage la forme (carrée), alors que vitre évoque le matériau (verre).

Nouv.PR

Ouvertures

HS

volet, contrevent $m$ shutter

Désigne un dispositif extérieur ou intérieur de fermeture de baie, composé de panneaux de bois, de métal ou de plastique.

Obs.: Autrefois les volets désignaient les fermetures intérieures alors que contrevent désignait les fermetures extérieures. L'anglais distingue inside shutter et outside shutter.

Ouvertures

HS

voligeage $m$ rough boarding

Ensemble de planches de bois légères qui consolide la charpente et l'aide ainsi à résister aux poussées latérales du vent.

Obs. : Par extension, les voliges, souvent de sapin ou de peuplier, clouées sur les chevrons, forment une surface sur laquelle la couverture sera clouée.

Toiture

JRS

voussure $f$ underside of a lintel

Face inférieure externe du linteau dans une baie.

Ouvertures

HS

zinc $m$ zinc

Métal gris-bleu pâle, cassant à froid, mais malléable à chaud.

Obs.: Peu sensible à la corrosion et durable, le zinc est, entre autres, un matériau de couverture appréciable. Cependant, le zinc doit rester à l'abri des fumées sulfureuses, du plâtre, de la chaux, du ciment, des acides et des bases. La tôle de zinc n'excède generalement pas $2 \mathrm{~m}$ de long. Sa largeur varie de 50 à $100 \mathrm{~cm}$. Toiture

JRS sill, window sill, outer sill principal rafter, sloping beam, top cord, top member

slate

hip

clay

asphalt

opening, aperture

Voir fenêtre panoramique shingle 


bardeau bitumé
barre inclinée
barre verticale
bâti dormant
battant
bédane
béton
béton armé
béton d'argile
béton gras
béton maigre
bétonnière
béton précontraint
biellette
bitume
bloc de ligne
blochet
bois
boîte à mélange
boîte de gâchage
boucharde
bouclier
brique
brique à angle
brique claveau
brique commune
brique creuse
brique de parement
brique de pavage
brique en coin
brique extrudée
brique ordinaire
brique perforée
brique pleine
brique réfractaire
brique sablée
briqueteuse
calcaire
cale
carreau
céramique
chambranle
chanlatte
charpente (de toit)
châssis
châssis à guillotine
châssis à la canadienne
châssis à la française
châssis à l'anglaise
châssis à l'australienne
châssis à l'italienne
châssis basculant
châssis coulissant
châssis de toiture
châssis inférieur
châssis pivotant
châssis supérieur
châssis-trémie
chaume
chaux

$\begin{aligned} & \begin{array}{r}\text { bitumen shingle } \\ \text { Voir diagonale } \\ \text { Voir montant } \\ \text { window casing }\end{array} \begin{array}{l}\text { chaux hydraulique } \\ \text { chaux vive } \\ \text { chevron } \\ \text { ciment } \\ \text { ciment à maçonner }\end{array} \\ & \begin{aligned} \text { cimement, àndow casement maçonnerie } \\ \text { mortise chisel } \\ \text { concrete }\end{aligned} \begin{array}{l}\text { ciment Portland } \\ \text { ciseau à bouchardage } \\ \text { ciseau à brique }\end{array} \\ & \text { reinforced concrete } \\ & \text { rammed clay } \\ & \text { fat concrete } \text { ciseau à dents } \\ & \text { lean concrete } \\ & \text { concrete mixer, cement mixer } \text { ciseau à froid } \\ & \text { prestressed concrete } \text { ciseau de maçon } \\ & \text { lever } \text { ciseau pinch } \\ & \text { bitumen } \text { clinker } \\ & \text { Voir coin } \text { coin }\end{aligned}$

tie beam piece, tie brace wood

mortar box, mortar pan

Voir boîte à mélange bush hammer

float, laying-on trowel brick angle brick

arch brick, compass brick,voussoir

Voir brique ordinaire air brick

facing brick, face brick paving brick

Voir brique claveau wirecut brick

building brick, common brick perforated brick, cored brick solid brick fire brick

sand-faced brick

Voir truelle briqueteuse limestone wedge Voir vitre ceramic, ceramics casing, architrave verge board

(roof) framing, frame, framework

casement, window casement, sash

Voir fenêtre à guillotine

Voir fenêtre à la canadienne

Voir fenêtre à la française

Voir fenêtre à l'anglaise Voir fenêtre à l'australienne

Voir fenêtre à l'italienne

Voir fenêtre basculante

Voir fenêtre coulissante

Voir fenêtre en toiture lower sash

Voir fenêtre pivotante upper sash

Voir fenêtre à soufflet thatch

Meta, XLII, 4, 1997

coin

comble

compression

contre-châssis

contre-fenêtre

contrefiche

contrevent

cordeau

cordeau à craie

cordeau d'alignement

cordeau traceur

couteau

couteau à mastic

couteau à mastiquer

couverture

coyau

crépissoir

croisée

croupe

diagonale

dormant

double fenêtre

double vitrage

égout

embrasure

enduit

entrait

entrait retrousse

équerre de maçon

faîte

faîtière (1)

faîtière( 2 )

faux-entrait

fendeuse

fenêtre

fenêtre à battants

fenêtre à guillotine

fenêtre à la canadienne

fenêtre à la française

fenêtre à l'anglaise casement, casement window, French casement fenêtre à l'australienne $\quad$ Austral window collar beam, collar tie outward-opening window

hydraulic lime quick lime rafter cement

masonry cement

Voir ciment à maçonner

Portland cement

pitching chise

claw chisel, claw tool,

tooth chisel, stonecutter's chisel

chip ax

Voir ciseau de maçon clinker roof structure compression

Voir contre-fenetre storm sash, storm window strut, stay, brace Voir volet

Voir ligne à briqueteur chalk line

Voir ligne à briqueteur

Voir cordeau à craie

Voir conteau à mastiquer putty knife roof-covering sprocket brush

Voir fenêtre hipped end diagonal

Voir bâti dormant double window

double glazed window, double glazing

opening, window opening

tie beam, main tie plumb rule ridge ridge-purlin

Voir panne faitière false tie beam

horizontal strut between principals Voir guillotine

Voir fenêtre à l' anglaise sash window, hung window Canadian sash, bottom-projected window 


fenêtre à l'italienne
fenêtre à soufflet
fenêtre à tabatière
fenêtre basculante

fenêtre coulissante fenêtre de doublage fenêtre de toit fenêtre en toiture fenêtre oscillo-battante fenêtre panoramique

fenêtre pivotante

fer
fer à joints

fer à plat

ferme

ferme à un poinçon

ferme classique

ferme complexe

ferme moderne

ferme simple

ferrer

feuillure

fibre-ciment

fil à plomb

finir les joints

flexion

gâcher

glaise

gouge

goutte d'eau

granit

granite

granulat

gratte

gratton

grès

grès cérame

guillotine

gypse

hachette

huisserie

isolation

isolation acoustique

isolation phonique

isolation thermique

jambage

jambe de force

jambette top-hung window, italian sash, top projected window bottom-hung window, hopper light, hopper vent skylight, rooflight horizontal pivoting window, horizontal pivot window, horizontal centre-hung window, tilting window sliding window Voir contre-fenêtre Voir fenêtre en toiture skylight, rooflight tilt and turn window picture window, window wall unit pivoting sash, vertically pivoting window, vertical pivoting window, pivoted window

iron jointer, joint tool, finishing tool, striking tool Voir langue-de-chat truss, roof truss Voir ferme simple [traditional truss] [complex truss] [triangulated truss] king post truss to fit locks and hinges rebate, rabbet cement fibre

plumb line, plumb bob, plummet to repoint, to tuck-point flexion to $\mathrm{mix}$, to temper Voir argile gouge Voir larmier granite

Voir granit aggregate mortar hoe devil float sandstone stoneware

masonry cutting machine, splitter gypsum hatchet

Voir bâti dormant insulation

Voir isolation phonique sound proofing, sound insulation

thermal insulation, heat insulation window post, jamb strut (of a truss), brace (of a truss), stay side post, princess post jauge

jauge à mesurer

jauge de briqueteur

jet d'eau

jointer

jointoyer

lame d'air

langue-de-chat

lantemeau

larmier

lattis

laye

liane

liant

ligne à briqueteur

linteau

lisseuse

liteau

malaxer

malaxeur à mortier

marbre

marne

marteau

marteau à brique

marteau de maçon

marteau pneumatique

martelet

masse de maçon

matelas d'air

matériaux

membrure inférieure

membrure supérieure

meneau

métal

minéral

mollon

montant (1)

montant (2)

montant battant

montant charnier

montant de battement

montant de rive

montant ferré

mortier

mortier bầtard

mortier de bourrage

mortier de chaux

mortier de ciment

mortier gras

mortier maigre

niveau à bulle

niveau de maçon

niveau à bulle d'air mortar
cement-lime mortar,

gauge rod, story pole, story rod

Voir jauge

Voir jauge

weatherboard, sill flashing

to point, to tool, to strike, to finish

Voir jointer airspace

calking trowel

skylight, lantern light

weather moulding,

drop apron, drip cap latice

axhammer, face hammer Voir lisseuse binder

mason's line lintel

plastering trowel, steel trowel splat

to $\operatorname{mix}$

mortar mixer, mortar mill marble marl

hammer

Voir marteau de maçon bricklayer's hammer, brick hammer jack hammer, pneumatic hammer tile hammer

sledge hammer

Voir lame d'air materials

bottom chord,

bottom member

top chord, top member mullion, transom (horizontal),

muntin (vertical) metal mineral rubble

upright, stile

upright, vertical member

Voir montant de battement

hanging stile

closing stile,

slamming stile

Voir montant charnier

Voir montant charnier mortar dry pack lime mortar cement mortar fat mortar lean mortar

Voir niveau à bulle d' air plumb rule spirit level 
noue

ouvrant

panne

panne à mortier

panneau

panneau de sous-toiture

panne faîtière

panne intermédiaire

panne sablière

parclose

paumelle

pente

pic

pièce d'appui

pied-de-roi

piédroit

pierre

pierre marbrière

pierre de taille

pierre à bâtir

pige

pignon

pince à brique

pistolet à calfeutrer

pivot

plâtre

plâtroir

plomb

poinçon

pointe

pointe de maçon

portée

poutre

pureau

rabot à mortier

rabot de maçon

rabotin

raclette

ragréer

ravaler

règle de maçon.

rejet d'eau

repère

rive

roche

ruban à mesurer de maçon

sablière

scie mécanique à maçonnerie valley

Voir châssis

purlin

Voir boîte à mélange

pane

Voir panneau

ridge beam, ridge piece,

ridge purlin

intermediate purlin

eaves purlin

glass stop, glazing bead,

glazing fillet

hinge

pitch

pick

bottom member

Voir jauge

Voir jambage

stone

marble-like stone

ashlar, cut stone

building stone

measuring rod

gable

brick clamps

caulking gun

pivot hinge

gypsum plaster

Voir lisseuse

Voir fil à plomb

king post

Voir ciseau de maçon

point chisel

span

beam

gauge

Voir rabot de maçon

rake, larry

drag, comb

scraper

to smooth

to rake

straightedge

Voir jet d'eau

screed

edge

rock

mason's rule,

steel tape

Voir panne sablière

masonry saw scie motorisée à maçonnerie Voir scie mécanique à

maçonnerie

scie sauteuse

set à brique

jigsaw, saber saw

Voir ciseau à brique

spatule

support à ligne

support de ligne

survitrage

tabatière

table à mortier

filling knife, broad knife

tingle

line twig

secondary glazing, overglazing

Voir fenêtre à tabatière

mortarboard,

tablette d'appui

mortar spot board, spot board, fat board

Voir appu

taloche

talocher

taloche souple

tamis

tavaillon

tendeur à ligne

théodolite

float, darby

to float

Voir lisseuse

sieve

Voir bardeau

Voir coin

theodolite roofing

toiture inclinée pitched roof

tôle

traction

traverse

traverse basse

traverse d'appu

traverse dormante

traverse haute

triangulation

truelle

truelle à bout carré

truelle à brique

truelle à jointoyer

truelle briqueteuse

truelle carrée

truelle d'angle

truelle de plâtrier

trumeau

tuile

valeur de recouvrement

vantail

verre isolant

vide joints

vitrage

vitrage isolant

vitre

volet

voligeage

voussure

zinc iron roof

traction

strut, crosspiece

bottom rail

Voir pièce d'appui

Voir linteau

top rail

triangulation

trowel

Voir truelle de plâtrier

brick trowel

pointing trowe

buttering trowel

Voir truelle de plâtrier

angle trowel

square trowel

pier

roofing value

Voir battant

Voir double vitrage

Voir bédane

glazing, window glazing

Voir double vitrage carreau, pane,

pane of glass, window pane shutter

rough boarding

underside of a lintel

zinc

Liste anglais-français

additive

admixture

aggregate

air brick

adjuvant

alloy

alliage

adjuvant aluminum

granulat amming stile

brique creuse angle brick

airspace

lame d'air, matelas d'air

angle trowel

montant de battement

brique à angle

truelle d'angle 


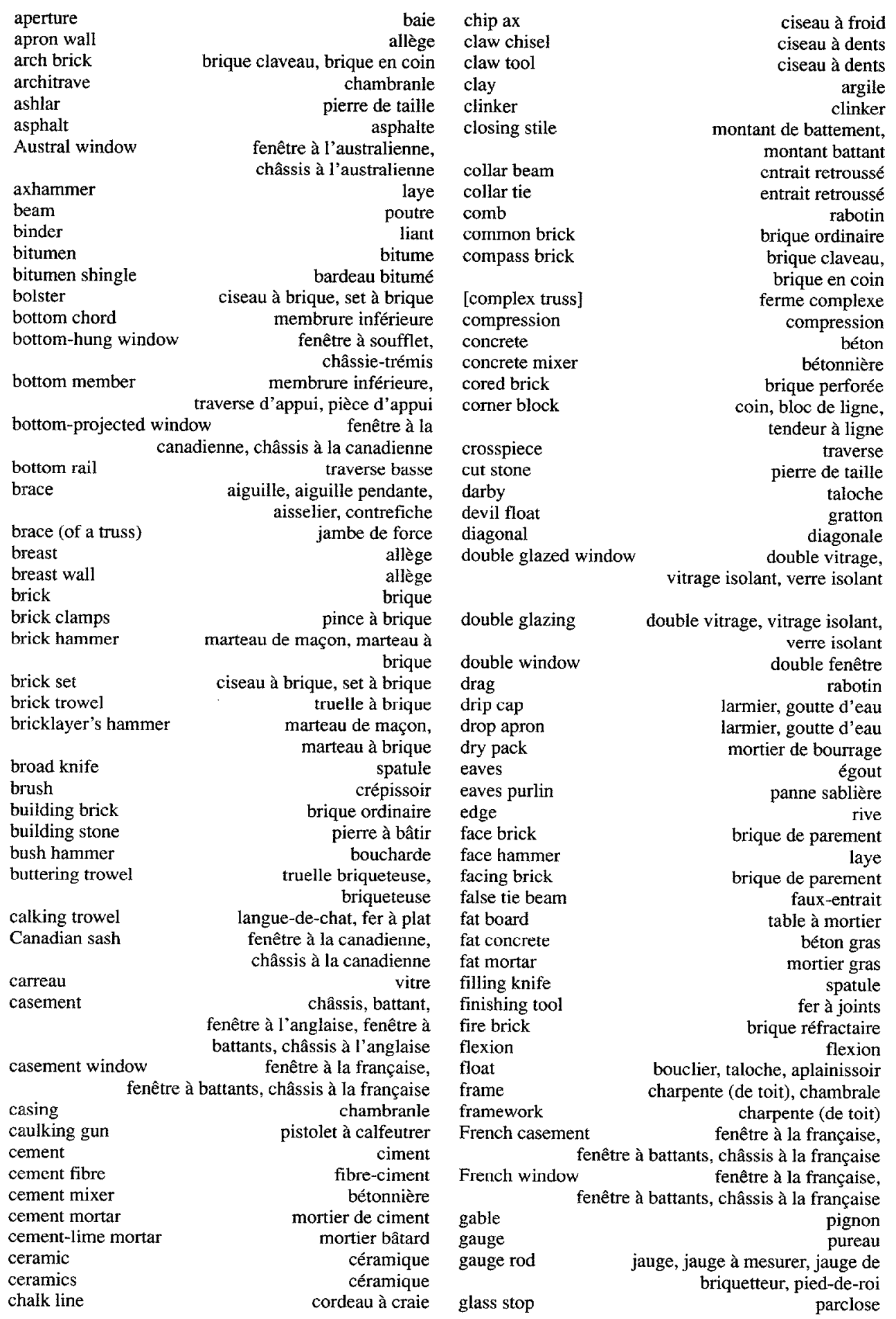




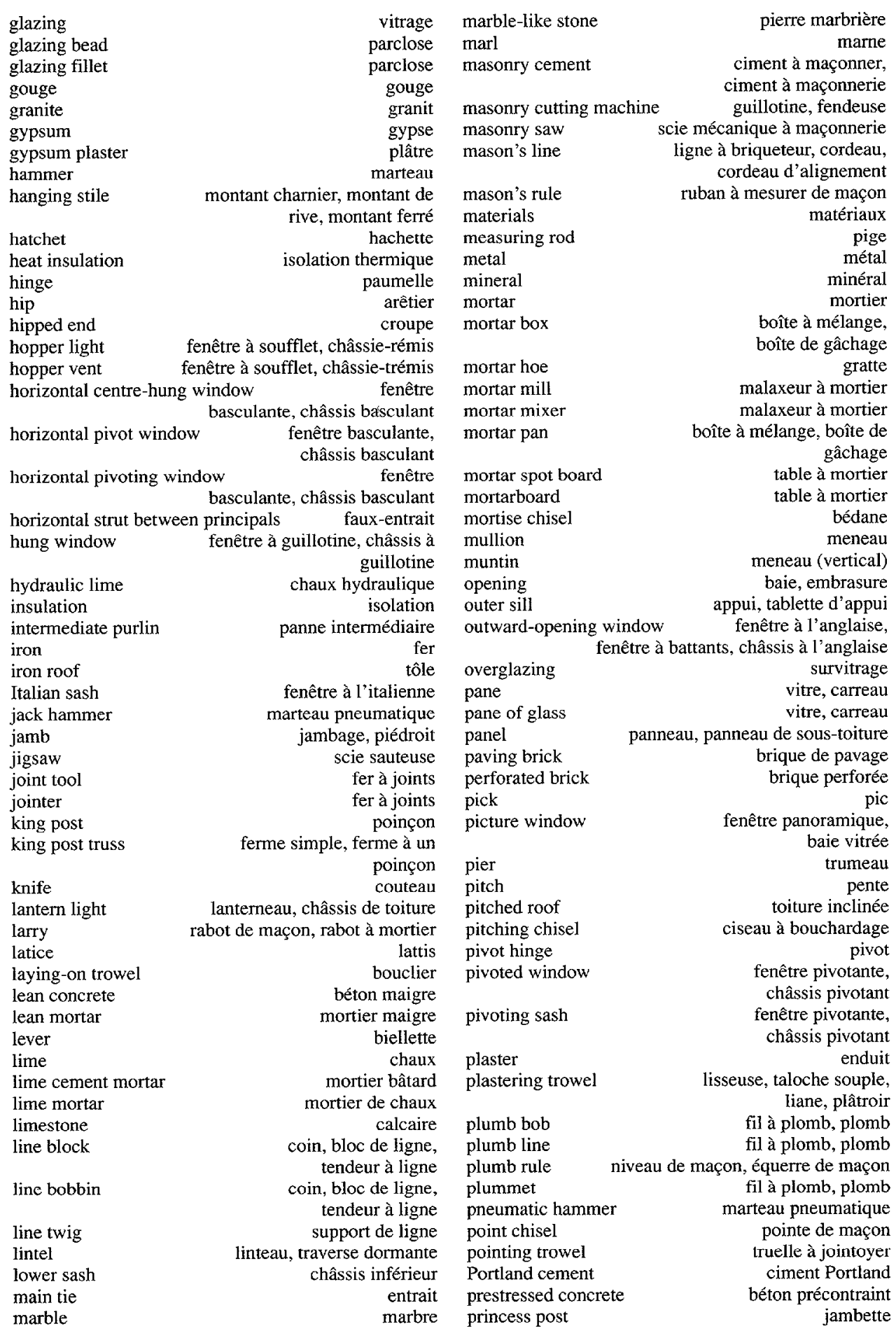




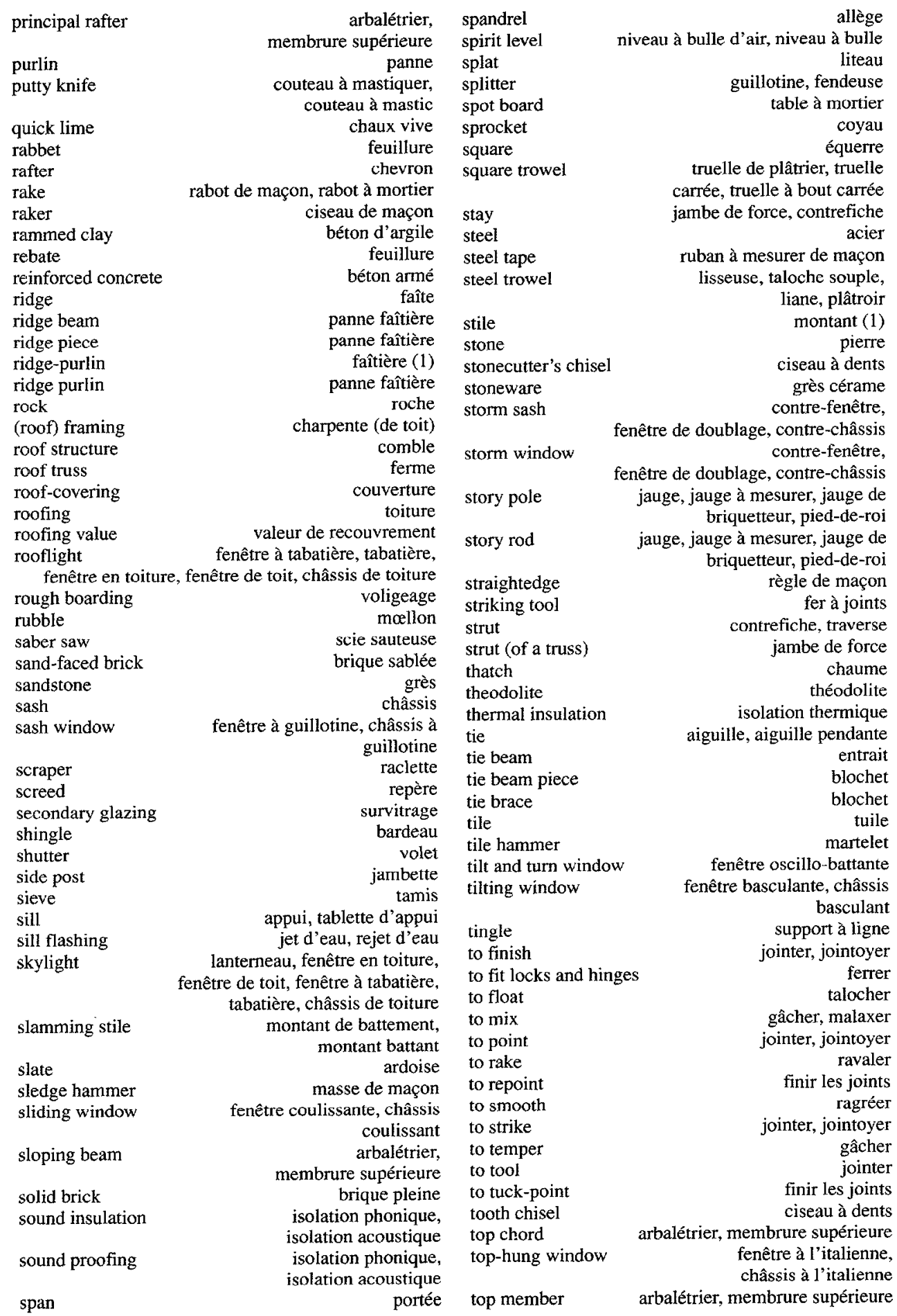


top projected window

top rail

traction

traditional truss

transom

[triangulated truss]

triangulation

trowel

truss

underside of a lintel

upper sash

upright

valley

verge board

vertical nember

vertical pivoting window

vertically pivoting window fenêtre à l'italienne, châssis à l'italienne traverse haute traction ferme classique meneau (horizontal)

ferme moderne triangulation truelle ferme voussure châssis supérieur montant (1), montant (2) noue chanlatte montant (2) fenêtre pivotante, châssis pivotant fenêtre pivotante, châssis pivotant voussoir weather moulding weatherboard wedge

window

window breast

window casement

window casement

window frame

window glazing

window lining

window opening

window pane

window post

window sill

window wall unit

wirecut brick

wood

zinc brique claveau, brique en coin larmier, goutte d'eau jet d'eau, rejet d'eau

$$
\text { cale }
$$

fenêtre, croisée

allège

battant

châssis

bâti dormant

vitrage

bâti dormant

embrasure

vitre, carreau

jambage, piédroit

appui, tablette d'appui

fenêtre panoramique, baie

$$
\text { vitrée }
$$

brique extrudée

$$
\text { bois }
$$

zinc 